\title{
Making predictable unpredictable with style - Behavioral and electrophysiological evidence for the critical role of prosodic expectations in the perception of prominence in speech
}

\author{
Sofoklis Kakouros ${ }^{\mathrm{a}}$, Nelli Salminen ${ }^{\mathrm{a}, \mathrm{b}}$, Okko Räsänen ${ }^{\mathrm{a}}$ \\ ${ }^{a}$ Department of Signal Processing and Acoustics, Aalto University, Finland \\ FI-00076, AALTO, Finland \\ ${ }^{\mathrm{b}}$ Aalto Behavioral Laboratory, Aalto Neuroimaging, Aalto University, Finland \\ FI-00076, AALTO, Finland
}

\section{Contact information:}

Okko Räsänen (corresponding author). Email: okko.rasanen@aalto.fi, GSM: +358-50-5911879. Dept. Signal Processing and Acoustics, Aalto University, P.O. Box 12200, FI-00076, AALTO, Finland.

Nelli Salminen, nelli.salminen@aalto.fi. Dept. Signal Processing and Acoustics, Aalto University, P.O. Box 12200, FI-00076, AALTO, Finland.

Sofoklis Kakouros, sofoklis.kakouros@aalto.fi. Dept. Signal Processing and Acoustics, Aalto University, P.O. Box 12200, FI-00076, AALTO, Finland. 


\begin{abstract}
Perceptual prominence of linguistic units such as words has been earlier connected to the concepts of predictability and attentional orientation. One hypothesis is that low-probability prosodic or lexical content is perceived as prominent due to the surprisal and high information value associated with the stimulus. However, the existing behavioral studies have used stimulus manipulations that follow or violate typical linguistic patterns present in the listeners' native language, i.e., assuming that the listeners have already established a model for acceptable prosodic patterns in the language. In the present study, we investigated whether prosodic expectations and the resulting subjective impression of prominence is affected by brief statistical adaptation to suprasegmental acoustic features in speech, also in the case where the prosodic patterns do not necessarily follow languagetypical marking for prominence. We first exposed listeners to five minutes of speech with uneven distributions of falling and rising fundamental frequency (F0) trajectories on sentence-final words, and then tested their judgments of prominence on a set of new utterances. The results show that the probability of the F0 trajectory affects the perception of prominence, a less frequent F0 trajectory making a word more prominent independently of the absolute direction of F0 change. In the second part of the study, we conducted EEG-measurements on a set of new subjects listening to similar utterances with predominantly rising or falling F0 on sentence-final words. Analysis of the resulting event-related potentials (ERP) reveals a significant difference in N200 and N400 ERP-component amplitudes between standard and deviant prosody, again independently of the F0 direction and the underlying lexical content. Since N400 has earlier been associated with semantic processing of stimuli, this suggests that listeners implicitly track probabilities at the suprasegmental level and that predictability of a prosodic pattern during a word has an impact to the semantic processing of the word. Overall, the study suggests that prosodic markers for prominence are at least partially driven by the statistical structure of recently perceived speech, and therefore prominence perception could be based on statistical learning mechanisms similar to those observed in early word learning, but in this case operating at the level of suprasegmental acoustic features.
\end{abstract}

Keywords: statistical learning, prosody, attention, prominence, event-related potentials, speech perception

\title{
1. Introduction
}

Stimulus predictability has an impact on our perceptual experience (see, e.g., Zarcone et al., 2016) and predictability-based accounts have explanatory power over many aspects of human language processing (see, e.g., Jurafsky, 1996; Jurafsky, Bell, Gregory, \& Raymond, 2001). For instance, all communicative signals, including words in speech, have to be at least somewhat unpredictable to the receiver in order to convey any information (Shannon, 1948). In addition, human perception employs highly efficient predictive models at multiple levels of representation (e.g., Grossberg, 1987; Friston \& Kiebel, 2009). In the context of speech, such predictive models allow listeners to overcome ambiguities in the input such as missing phonemes or ambiguously pronounced words by constraining the set of potential messages in the given context (e.g., Warren, 1970; Samuel, 1987; 
see also Federmeier, 2007, or Zarcone et al., 2016, for a discussion). Although the main purpose of communication is to alter the internal state and memory of the listener, the presence of such predictive models also means that previous learning and the current state of the listener's cognitive system have an impact on what and how things are perceived. In terms of general perceptual processing, this type of interaction between top-down ${ }^{1}$ predictions and bottom-up sensing is a useful strategy, as it enables a more complete representation of the environment than what would be available purely based on the currently unfolding sensory input.

Top-down predictions also enable more efficient use of the brain's computational resources, as resources for sensing and learning can be allocated to those aspects of the environment that are not yet modeled (novel) or are otherwise rare (surprising) and thereby not anticipated by the system (see, e.g., Ranganath \& Rainer, 2003). In terms of information theory, selective processing of less predictable inputs leads to higher information gain in learning as long as the stimulus and its context are sufficiently structured to provide consistent predictive associations in the future (cf. the Goldilocks-effect where infants tend to attend to stimuli that are neither too simple nor too complex; Kidd, Piantadosi \& Aslin, 2012, 2014). In the end, it makes sense to prioritize learning from experiences that differ from the expected outcomes for any given situation, especially when learning and sensing have metabolic, computational, or opportunity costs associated with them.

Selective processing of sensory input inherently connects to the concepts of attention and prominence: within a predictability-based framework, stimulus-driven attention can be viewed as a process where surprising aspects of the sensory input receive high-priority sensing and cognitive processing due to their highest information value for the agent (see also Zarcone et al., 2016), which then results in a subjective impression of prominence (or saliency) in contrast to baseline stimuli due to the capture of attentional focus. For instance, visual attention and statistical predictability of natural stimuli have been shown to be connected, less predictable stimuli acting as stronger magnets for visual fixations (Itti \& Baldi, 2009; Zhang, Tong, Marks, Shan \& Cottrell, 2008). There is also evidence that saliency of auditory events is more correlated with the predictability in the statistical structure of sounds than their specific feature values as such (Tsuchida \& Cottrell, 2012). Informally, aspects of an otherwise stable environment that are surprising to us also tend to capture our attention, whereas familiar environments and routine patterns require little or no conscious effort or attentional focus.

The concept of prominence is also central to the domain of speech communication and language acquisition. Prominence in speech refers to the phenomenon where certain parts of the speech signal are perceptually standing out from their context (e.g., Terken, 1991). This is usually associated with the introduction of new or otherwise significant information with respect to earlier discourse and the existing assumed knowledge of the listener (see, e.g., Bock \& Mazzella, 1983; Terken \& Nooteboom, 1987). Although prominence in speech is often approached from a linguistic point of view and its definitions vary (see Wagner et al., 2015, for a discussion), earlier work has also connected prominence in speech with the predictability at the acoustic and lexical levels (e.g., Cole, Mo \& Hasegawa-Johnson, 2010; Aylett \& Turk, 2004; 2006; Pan \& McKeown, 1999; Pan \&

\footnotetext{
${ }^{1}$ In the present context, we use the term top-down predictions to refer to temporal or cross-sensory predictions emerging from the current representational state of the cognitive system, not to task-driven executive processes and their consequences on perceptual processing.
} 
Hirschberg, 2000; Watson, Arnold \& Tanenhaus, 2008). For instance, the Smooth Signal Redundancy Hypothesis of Aylett and Turk (2004) treats acoustic prominence as a result of information rate optimization at a linguistic level, while others have used lexical predictability as a useful cue for automatic prosodic marking (e.g., Pan \& McKeown, 1999; Pan \& Hirschberg, 2000). Buxó-Lugo and Watson (2016) have shown that expectations primed by the syntactic context affect interpretation of acoustic cues for intonational boundaries. Cole et al. (2010) demonstrated that listeners' judgments of prominence in words are dependent on both lexical predictability and on the acoustic characteristics of stressed vowels in the same word. This led Cole et al. (2010) to suggest that prominence in speech could be partly driven by the acoustic features of the signal and partly by listener's expectations at the lexical level, both leading to capturing of listener's attention. Bishop (2012) also showed that the context-dependent information structure of an utterance has an impact on the perception of prominence for the words in the utterance. However, these existing theories and studies focus on examining the predictability of linguistic units (e.g., phonemes, words) and how this impacts the acoustic realization of the units and the concurrent prosodic patterns. In contrast, relatively little is known about how the predictability of the low-level or suprasegmental acoustic features affects the interpretation of the linguistic units themselves.

In our earlier work, we suggested that the acoustic component of prominence could also be expectation driven instead of being based on specific acoustic feature configurations as such (Kakouros \& Räsänen, 2016a). We also argued that models trying to explain prominence purely as a result of linguistic structure and constraints cannot capture the full breadth of the phenomenon. This is because the talker can always freely (but not necessarily consciously) choose which word to emphasize (see also Bolinger, 1972; 1983), either using a less expected word choice (lexical predictability) or a non-expected suprasegmental articulation (prosodic predictability) on any given word. The talker can thereby tap to the listener's attentional mechanisms by surprising the listener, triggering non-canonical processing of the emphasized content, possibly enhancing learning or leading to broader semantic interpretation of the message. The listener, on the other hand, has to rely on the a priori expectations for the word choices and acoustic prosodic outcomes that are most typical in the context, given the previous experience with the language and the talker in question. By violating these expectations using modifications at the segmental or suprasegmental level, the talker can trigger the listener's generic mechanisms for perceptual prediction and attentional orientation. For instance, contrastive stress patterns in utterances "I went home", "I went home", and "I went home" are all valid prosodic constructs, but still carry different semantic implications to the listener. Importantly, even if the stress falls on a word that is "expected" to carry stress in a given context (i.e., given the information structure of the discourse), the listener does not generally have access to the exact information structure intended by the talker before the sentence unfolds in time, necessarily introducing uncertainty to the occurrence of stressed words in real-time comprehension.

Importantly, this type of fully probabilistic formulation of prominence does not dictate any specialized cognitive mechanisms or specific acoustic feature detectors in prominence perception. Instead, a general statistical learning mechanism ${ }^{2}$ paired with attentional preference for unfamiliar

\footnotetext{
${ }^{2}$ In the literature on learning, a distinction between the so-called distributional learning (e.g., Maye et al., 2002) and statistical learning (e.g., Saffran et al., 1996) is sometimes made, the former referring to the human ability to learn
} 
events (cf., Saffran, Aslin \& Newport, 1996; see, e.g., Aslin \& Newport, 2012, or Erickson \& Thiessen, 2015, for overviews) operating at prosodic and linguistic levels would be sufficient to generate the necessary interaction between bottom-up signal contents and experience-based topdown predictions. This would also explain how language-specific cues (section 1.1) to prominence are acquired without formal training and how listeners can rapidly adapt to talker-specific idiosyncrasies in speech. Probabilistic interpretation of prominence can also have important consequences to understanding the basis of infant-directed speech (IDS) since IDS is believed to have a role in directing and maintaining the infants' attention towards certain aspects of the perceptual experience, i.e., controlling the attention of listeners who are adept statistical learners but do not yet master the language in question and therefore lack capability to analyze prosodic patterns in terms of linguistic function (see, e.g., Cooper \& Aslin, 1994, and references therein).

In support of the acoustic predictability hypothesis, we have earlier shown that unpredictability of acoustic prosodic trajectories is highly correlated with human judgments of prominence on the same input in several different languages (Kakouros \& Räsänen, 2014, 2016a, 2016b) and that both prosodic and lexical predictability contribute to the prominence of words (Kakouros, Pelemans, Vervimp, Wambacq \& Räsänen, 2016), effectively replacing the signal-based component of Cole et al. (2010) with a fully probabilistic model at multiple levels of representation. The hypothesis also receives support from electrophysiological studies. For instance, an earlier electroencephalography (EEG) study by Magne et al. (2005) shows that violation of languagetypical accentuation patterns leads to changes in the event-related potential (ERP) component N400 that is associated with semantic processing of the input. Notably, this effect was found to be independent of the actual presence or absence of acoustic cues for accentuation in French, but instead simply depended on whether the presence or absence of accentuation was expected in the given discourse context. The study by Magne et al. (2005), together with many other EEG studies with similar findings (see section 3.1), indicates that the preceding linguistic context interacts with prosodic expectations for incoming speech, and that violations to these expectations lead to altered semantic processing of the underlying words, at least when the accentuation follows or violates regularities typical to the native language of the listeners. In general, there is an increasing number of electrophysiological evidence for the role of predictive processing at the level of audition (see Bendixen, SanMiguel, \& Schröger, 2011, for a review).

However, based on these earlier studies, it is still unclear how the prosodic expectations come into place and whether the relationship between prosodic predictability and prominence is more correlational than causal in nature. The present study attempts to address these issues by first testing whether listeners' perceptual cues to prominence can be manipulated by exposing them to specific distributions of prosodic trajectories while carefully controlling for every other aspect of the stimuli and then asking the subjects to mark prominent words in utterances with similar manipulations. In the second part of the study, we use a similar habituation paradigm with

(statistical) regularities across a number of measurable sensory input dimensions, while the latter is often used in the context of learning temporal patterns such as transition probabilities between syllables. In the current paper, however, we will refer to both aspects simply as "statistical learning". This is because both can be interpreted as learning of above-chance statistical patterns from the perceptual input where distributional learning can be framed as a (simplified) "zeroth-order" temporal model, i.e., where predictability of the input is not dependent on the surrounding context. 
concurrent EEG measurements to explore the neural underpinnings of perceptual processing of words during high- and low-probability prosodic patterns.

\subsection{Earlier work on prominence and predictability}

Prominence is a prosodic phenomenon that can be generally defined as the property by which linguistic units are perceived to be standing out from their environment (Terken, 1991; see also Cutler, Dahan, \& van Donselaar, 1997; Shattuck-Hufnagel \& Turk, 1996, for related definitions). As the terminology can be ambiguous, we will use the word prominence throughout this paper when referring to the perceptual impression of emphasis on specific words in speech. In contrast, the term stress will be used to refer to the production aspect of the phenomenon where a speaker intends to emphasize some units over some others. Note that speakers can stress words in order to make them more prominent, but the listeners perceive some words as more prominent than some others due to a number of factors that are not necessarily limited to the intended emphasis of the talker. In addition, stress can be realized phonetically in several different ways.

In terms of the acoustic correlates of prominence in speech, it is well established that energy, fundamental frequency (F0), duration (see, e.g., Fry, 1955, 1958; Lieberman, 1960; Terken, 1991; Kochanski, Grabe, Coleman, \& Rosner, 2005; see also Ortega-Llebaria \& Prieto, 2010, and references therein), and spectral tilt (see, e.g., Sluijter \& van Heuven, 1996; but see also Campbell, 1995; Campbell \& Beckman, 1997) are the acoustic parameters whose variations signal prominence and constituent boundaries (see also Shattuck-Hufnagel \& Turk, 1996). Beyond the physical acoustic correlates, prominence has been examined from a number of different perspectives (see, Wagner et al., 2015, and references therein). For instance, from a functional perspective, prominence has been studied with respect to its linguistic and communicative role. The realization of prominence seems to have effects on the parsing of information and syntactic structure of utterances (Calhoun, 2010; Shattuck-Hufnagel \& Turk, 1996). In addition, reaction times (RTs) for prominent words have been found to be shorter when compared to their non-prominent counterparts (see, e.g., Cutler \& Foss, 1977) while accentuation has been shown to improve detectability of lexical changes between two similar utterances (Sanford et al., 2006). Prominent words also seem to attract the listener's attention, thus allocating extra cognitive processing resources (see, e.g., Cole, Mo, \& Hasegawa-Johnson, 2010).

Since all speakers are equipped with the same production and perception apparatus, the ways of conveying information through speech, including markers for prominence, should not vary greatly between languages (Vaissière, 1983). However, the actual realizations of prosodic patterns still have language-dependent components (see, e.g., Rosenberg et al., 2012; Maier et al., 2009), especially when some of the acoustic prosodic features are also utilized to signal phonemic contrasts, e.g., F0 in tonal languages such as Mandarin Chinese (see, e.g., Moore, 1993) or unit durations in languages with durational quantities such as Finnish (see, e.g., Suomi, Toivanen \& Ylitalo, 2008). Dialects of the same language can also vary in their realization of prominence (e.g., Ylitalo, 2009), and ultimately any information on potential talker- or context-specific differences in signifying prominence are largely lost in the population-level aggregate measures used in typical studies. In total, although the realization of prominence is necessarily constrained by the biomechanical constraints of the universally shared speech apparatus, it also has a component based 
on local communicative conventions and experience with the linguistic community. This also means that the language learners and users must be equipped with some kind of learning mechanisms that are sensitive to prosodic patterns in speech.

One potential mechanism for such learning is the so-called statistical learning mechanism (also distributional learning), a generic term referring to infant and adult capability to track probabilistic regularities in sensory experience. Statistical learning originally referred to infants' capability to learn high-probability syllabic sequences from artificial languages (Saffran et al., 1996) or distributions of acoustic features (Maye, Werker \& Gerken, 2002), but is now known to be a much more general cognitive process that spans beyond specific linguistic units or sensory modalities (see, e.g., Aslin \& Newport, 2012, or Erickson \& Thiessen, 2015, for overviews). Importantly, statistical learning is often studied using experiments that are based on selective attention of the subjects (infants or adults) in controlled conditions (e.g., eye-tracking or preferential head-turn paradigm), inherently coupling the measurement of statistical learning with attentional processing. Since statistical learning seems to operate across various levels of representation, it would be surprising if the same mechanism tracking regularities across syllables or non-linguistic segments such as tones (Saffran, Johnson, Aslin \& Newport, 1999) would not also operate on the suprasegmental level of speech, especially when both segmental and suprasegmental components are merged into one holistic acoustic signal that, for instance, infants learn to decode into its linguistic constituents only gradually.

However, according to our knowledge, no existing work has systematically explored statistical learning at the suprasegmental level using continuous speech while controlling for the underlying lexical content, testing whether the relationship between suprasegmental predictability and impression of prominence is more correlational or causal in nature (cf., Kakouros \& Räsänen, 2016a). It is also unknown whether the acoustic cues can be unpredictable in general, or whether they still have to follow certain linguistic conventions for stress in order to play the same functional role as "normal" emphasis on words. Finally, it is unclear how long-term language experience (cf., e.g., Magne et al., 2005) and recent short-term exposure with different statistics interact in creating expectations at the suprasegmental level.

The present study attempts to shed some light on these issues. In experiment 1, we test short-term adaptation to the statistical properties of prosodic patterns by exposing listeners to controlled distributions of prosodic trajectories and then asking the subjects to annotate a set of new utterances for prominent words with similar prosodic manipulations. In experiment 2, we measure event-related brain responses for words with prosodic manipulations using EEG in order to investigate whether prosodic probabilities, but not absolute physical properties, also impact neural signatures of speech parsing and whether probabilities of prosodic trajectories interact with ERPcomponents previously associated with semantic analysis of language input.

\section{Experiment 1: Statistical learning of prosodic trajectories}

The goal of the first experiment was to investigate whether a brief exposure to a certain distribution of rising and falling F0 trajectories on sentence-final words affects listeners' perception of prominence in utterances following the exposure. The hypothesis arising from the predictability 
framework (Kakouros \& Räsänen, 2016a) was that words during low-probability F0 patterns, that is, the patterns that are less frequent during the habituation stage, would be regarded as more prominent than exactly the same patterns when they are more frequent in the habituation, independently of the actual direction of the F0 change during the word. If true, this would suggest that listeners are tracking stimulus probabilities at a suprasegmental level and that these probabilities are related to subjective impression of prominence. In addition, this would mean that a brief exposure to a certain statistical distribution can override the baseline model the listeners may have from their lifelong experience with their native language.

\subsection{Methods for experiment 1}

\subsubsection{Experimental design}

Our target was a fully counter-balanced experimental design where the only independent variable in the population mean responses for the two compared conditions would be the predictability of the F0 trajectory, one condition having low-probability F0 trajectory (deviant condition) while the other having high-probability F0 (standard condition) while all other properties of the signals would be exactly the same between the two conditions. In order to achieve this, we divided our test subjects into two groups: falling F0 standard condition (FSC) and rising F0 standard condition (RSC). The former group (FSC) heard four-word utterances with a flat F0 trajectory up to the onset of the last word in the utterance, and then falling F0 in 90\% and rising F0 in 10\% of the sentence-final words. The RSC group heard exactly the same set of target utterances, but now with $90 \%$ sentence-final words having a rising F0 while the remaining 10\% had a falling F0 (Fig. 1). After listening to a stream of such sentences for approximately 5 minutes, the listeners were given a task to annotate prominence on a number of new four-word utterances with similar F0 manipulations on sentencefinal words, and we measured the average prominence ratings for the manipulated words.
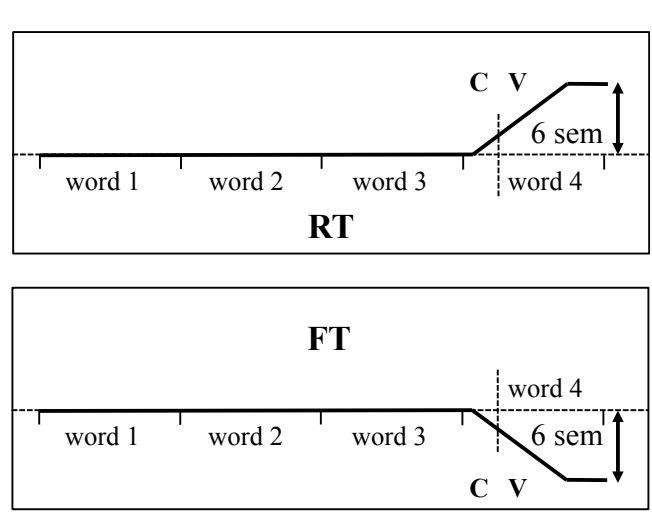

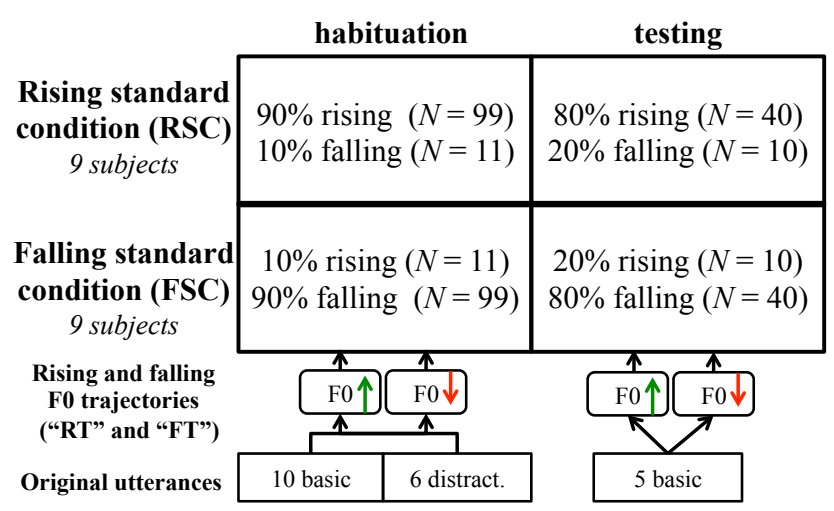

Figure 1: Left: F0 trajectories for the two conditions. Top panel: contour for the rising trajectory (RT), Bottom panel: contour for the falling trajectory (FT). The dashed vertical line represents the first vowel onset of the sentence-final word. Right: Overview of the experimental setup.

\subsubsection{Stimuli}

Speech samples from the CAREGIVER Y2 FI corpus (Altosaar et al., 2010) were used in the study. The style of speech in CAREGIVER is enacted infant-directed speech (IDS) spoken in continuous Finnish, corresponding to a situation where a caregiver is talking to a child, and recorded in high 
quality in a noise-free anechoic room. The corpus was selected due to the availability of multiple sentences with a simple subject-verb-object (SVO) syntactic structure and regular speaking rate that allows easier manipulation of the F0 patterns in the sentences. The reading scripts for utterances were originally generated by combining grammatical templates ("carrier sentences") with randomly sampled nouns, verbs, and adjectives from a finite lexicon in order to have statistically balanced combinations of words with grammatically correct sentences, and hence leading to a range of utterances from semantically coherent to atypical combinations of adjectives and nouns (see below; see also Altosaar et al., 2010, for details). 21 unique utterances from a female speaker (Speaker 2) were chosen for the experiments based on the overall naturalness of the stimuli after manipulating the F0 trajectories of the utterances. Each stimulus consisted of a four-word SVO sentence with an average duration of three seconds.

Ten of the utterances were assigned as the main habituation stimuli and five utterances were used solely for the testing stage, all having semantically plausible contents such as "He sees a big animal" (translated). The remaining 6 utterances formed a so-called distractor set. More specifically, an overt task was utilized to ensure that the subjects were engaged in listening to the speech input during habituation, but without explicitly asking them to focus on the prosodic characteristics of the input. In this semantic decision task, the subjects were instructed to listen to the utterances and press a button whenever they heard a semantically incongruent sentence. These distractor sentences, such as "The baby gives the square dog" (translated), were original productions from the CAREGIVER corpus that had semantically anomalous adjective-verb pairs. The distractor sentences were interleaved randomly in the data, covering a total of $1 / 11$ of all utterances. Each distractor had a falling and rising F0 version, and their F0 trajectories followed the same 9:1 standard-deviant ratio as our actual target sentences. All button presses from the subjects were recorded to ensure that they paid attention to the experiment, and one of the debriefing questions was aimed to make sure that they had understood the task properly. Distractors were not included in the data analyses.

For each of the 21 utterances, we generated two prosodic versions with either a falling F0 trajectory (FT) or rising F0 trajectory (RT) on the last word using the pitch manipulation functionality available in the Praat software (Boersma \& Weenink, 2012). A rising F0 during the initial syllable, followed by a falling F0 on the later syllables of a word, is typically used to signal accent in Finnish spoken language where the primary stress always falls on the first syllable (Suomi \& Ylitalo, 2002; Suomi, Toivanen \& Ylitalo, 2008). However, due to the inherent variability in the number of syllables in the target words and our aim to have exactly the same acoustic manipulations for each word, we chose to model only the rising or falling F0 during the word-initial syllables as this was sufficient to cause a perceptually satisfactory impression of accentuation. For each stimulus, the original F0 contour was first flattened to $185 \mathrm{~Hz}$ (approximately the average across the original stimuli), reflecting the most natural sounding fixed pitch for the speaker. The F0 trajectory was then modified for the fourth word ("target word") of each stimulus while keeping the rest of the contour flat (Fig. 1). The pitch excursion for the falling and rising trajectories was set to start right before the vowel onset of the first syllable of the fourth word (see, e.g., Hermes \& Rump, 1994). To ensure consistency, the pitch excursion for all stimuli started 50-ms before the vowel onset and peaked 150-ms later, staying constant for the remaining part of the utterance (Fig. 1), 
thereby approximately matching to the onset part of the F0 excursion data reported in Suomi et al. (2008).

As the relation between pitch excursion size and prominence perception may vary, some studies reporting that a difference of 1.5 semitones (Rietveld \& Gussenhoven, 1985) or even 4 semitones (Hart, 1981) is required for a perceptually noticeable difference, we preliminarily experimented with a number of different excursion sizes. After assessing excursion sizes between 2 and 8 semitones, we selected a 6-semitone change from the flat F0 as the difference producing a clear and most natural-sounding perception of prominence across all stimuli. Thus, the modified trajectories during the target words had \pm 6 semitone excursion from the flat $185 \mathrm{~Hz} F 0$ of all previous words in the utterance (Fig. 1). After resynthesizing the utterances with the modified F0 trajectories, all stimuli were amplitude normalized.

Overall, the flat F0 during the first three words and a fixed rising/falling ramp on the final word was seen as a reasonable tradeoff between control over the F0 and naturalness of the stimuli. Relatively flat or, more commonly, monotonically falling F0 contours are often encountered in conversational Finnish where intonation is not used for signaling word stress (but still used for sentence stress), and where accentuation plays only a limited syntactic role in comparison to Germanic languages such as English and Swedish. In addition, accentuation, when present, tends to fall on the final word of a rhythm group similar to the current stimuli (see Suomi et al., 2008). Despite the fact that artificial flattening of the F0 contours necessarily introduces some speech quality and timbre changes due to a non-ideal separation of the voice source from the rest of the vocal tract, we felt that the F0 manipulations were sufficiently subtle to keep the listeners' focus on the speech content instead of the form. In support of this assumption, none of the listeners mentioned sound quality or artifacts after the experiment but all post-test comments were focused on semantic congruency of the stimuli.

\subsubsection{Participants}

Twenty $(N=20)$ native Finnish speakers (average age $28.4 \pm 3.8$ years; 13 male) participated in the listening experiment. The test subjects were recruited from the personnel and students of Aalto University. All participants reported normal hearing. The subjects were initially randomly assigned to the two test conditions. Data from two subjects had to be discarded due to 1) a technical problem in measuring button presses, and 2) inability of the subject to understand the task as self-reported during debriefing. Both subjects were from the RSC group and this was taken into account in the assignment of the last measured subjects into groups, leading to a total of 9 subjects per condition.

\subsubsection{Experimental procedure}

The listening experiment was conducted in a sound-isolated listening booth of the Acoustics Laboratory of the Aalto University. The habituation and testing software was run on a Mac mini with Matlab 2014b. The audio from the computer was fed through a Motu UltraLite-mk3 Hybrid into a pair of high-quality Sennheiser HD650 headphones.

Participants were given a brief description of the task by the experimenter and they were then asked to start the experiment. The experiment consisted of two parts: (i) a habituation and (ii) a testing stage (see Fig. 1 for an overview). During the habituation stage, participants were asked to 
listen carefully to each utterance being played and press the spacebar whenever they heard a semantically incoherent sentence (the distractor). The role of the overt task was to ensure that the subjects engaged into holistic lexical and semantic processing of the stimuli during habituation. The subjects were not given specific instructions regarding what counts as a semantically incoherent target, but were instructed to use their own judgment.

The only difference between the subject groups, that is, the RSC and FSC conditions, was the distribution of the rising and falling F0 trajectories during the habituation and testing stage. In the case of RSC, $90 \%$ of the stimuli were RT ("standards") and 10\% were FT ("deviants") while the situation was opposite for the FSC condition. Six semantically incoherent targets (distractor stimuli) were interleaved in the data and also followed the same 9:1 ratio. Each participant in each condition heard each lexically unique training utterance exactly the same number of times: nine times with standard F0 and once with deviant F0. In addition, they heard each distractor once. The total duration of the habituation stage was approximately 5 minutes, corresponding to a total of 110 utterances with a 500-ms silent interval between utterances. To avoid repetition of the same utterance in a sequence, the stimuli were presented in 10 blocks of 11 utterances. Each block contained the 10 unique training sentences (nine standards, one deviant; Fig. 1) and one distractor, all in a pseudo-randomized order within each block (different order for different subjects). The deviant target utterance in each block was different (i.e., all lexically unique utterances occurred once as the deviant across the whole familiarization), nine of the blocks had a distractor with standard F0, and one randomly chosen block had a distractor with a deviant F0 contour. Ordering of the blocks was also pseudo-randomized separately for each subject, with the constraint that the first sentence of a block had to be different from the final sentence of the previous block. There were no audible pauses between the blocks.

In the second part of the experiment, the subjects heard the test utterances one-by-one and, for each utterance, they were asked to select the single most prominent word and grade its prominence level on a nominal scale of $1=$ slight prominence and $2=$ notable prominence, or 0 if none of the words were perceptually prominent. Subjects were allowed to hear each utterance only once in order to capture initial perceptual impressions and to ensure controlled distribution of the pitch trajectories during the testing. The tasks of word selection and prominence grading were carried out using a graphical user interface where a list of the spoken words was presented together with the prominence scale. Subjects used a mouse as the controller to make the selection. The stimuli distribution in the test stage was set to have $80 \%$ of standards (RT for RSC; FT for FSC) and $20 \%$ of deviants (FT for RSC; RT for FSC) in order to obtain more test samples for the deviants than what would be available from the original habituation distribution. The stimuli were presented in blocks of 5, each comprising of four standards and one deviant F0. There were 10 blocks of test stimuli, adding up to a total of 50 test tokens per subject and corresponding to approximately 15 minutes to complete, on average. There were no distractor stimuli during the testing stage.

Overall, each subject heard exactly the same set of non-distractor training utterances (the same lexical content) exactly the same number of times during habituation $(N=100)$, and another set of similarly balanced and previously unheard utterances $(N=50)$ during the testing stage. In addition, each lexical target utterance and its two prosodic variants were used as standards and 
deviants equal number of times across the two subject groups, F0 being always fully independent of the lexical content. This left the probability (but not direction or lexical content) of word-final F0 to be the only differentiating factor in the averaged prominence ratings reported in the next section.

After the experiment, the subjects were debriefed with two general questions: 1) whether they had any general comments about the experiment, and 2) whether they had any comments regarding the stimuli.

\subsection{Results}

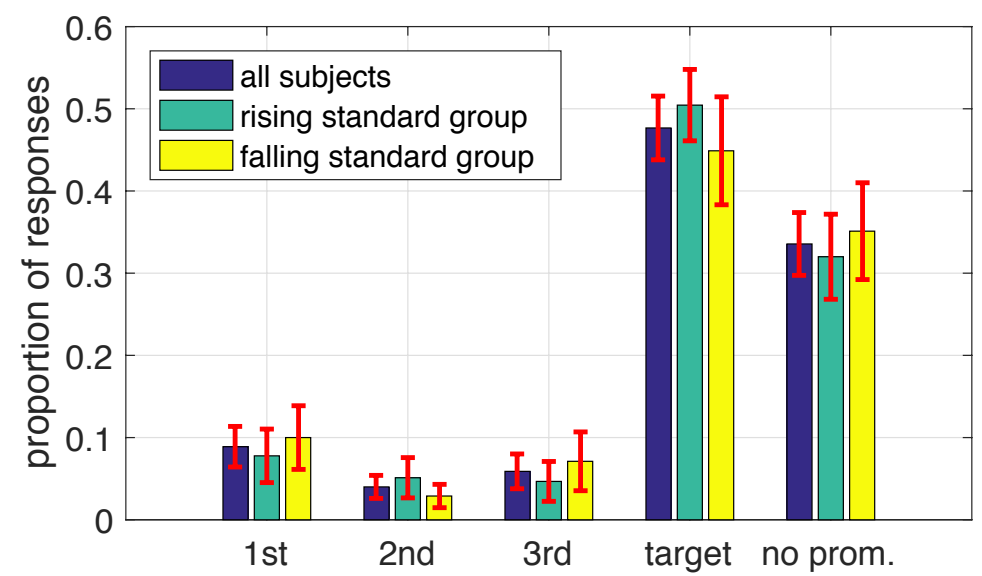

Figure 2: Proportion of responses to different words in the 4-word test sentences (relative position), including the no-prominence option. The error bars denote one standard error (SE) of the mean.

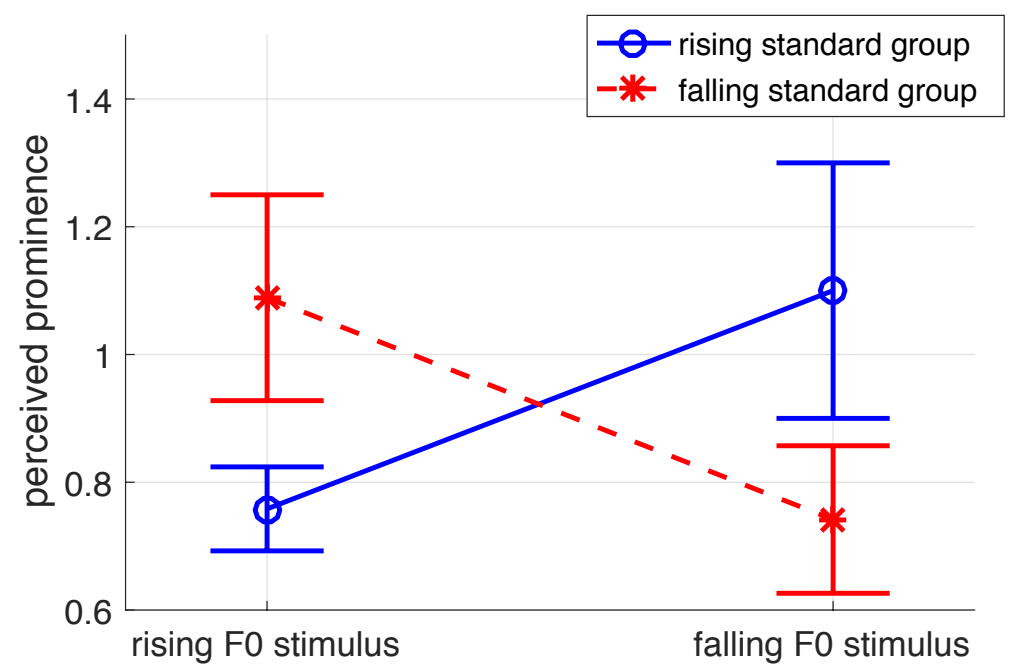

Figure 3: Perceived prominence for both subject groups and for both stimulus types (RSC with blue solid line and FSC with red dashed line). The error bars denote one standard error (SE) of the mean. 

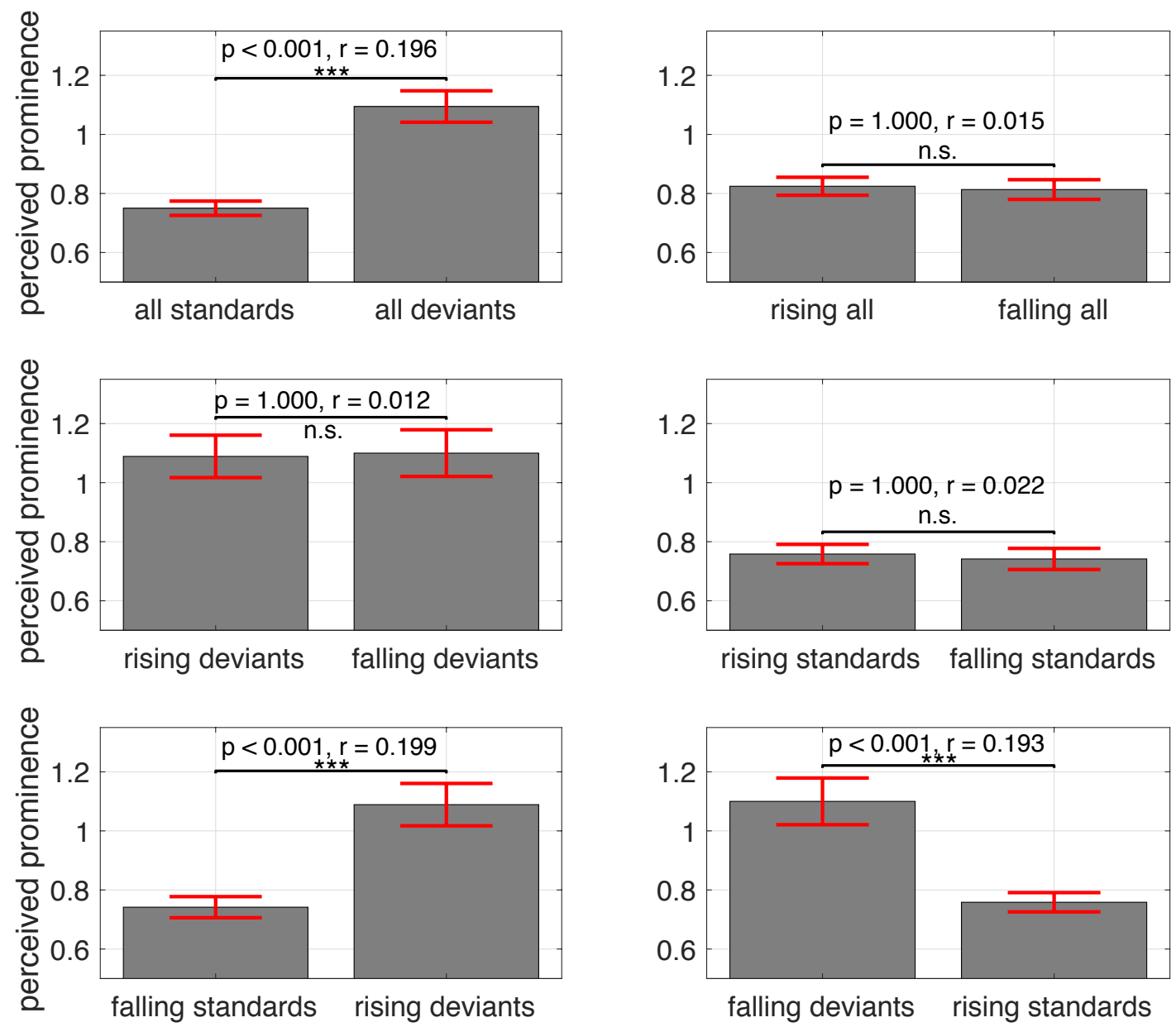

Figure 4: Means and SEs for the perceived prominence (0-2) levels for different stimulus types. The main comparison is shown on top left. Significance and effect sizes are reported using the Wilcoxon rank-sum test and using Bonferroni corrected significance level for $\mathrm{p}<0.05$ for the six post-hoc comparisons.

We first verified how often the subjects labeled utterance-final words, the targets, as prominent instead of other words in the sentences. Fig. 2 shows the proportion of responses to each of the four words (relative positions) in the test sentences and for no-prominence option for samples pooled across all subjects. The subjects primarily considered the manipulated target word as a prominent or no word as prominent with less than 19\% of the responses marking one of the first three words in the utterances as prominent. In addition, the response strategies between the two subject groups were similar with the same proportion of responses to the targets $(p=0.491, t=$ 0.706, $d f=16$, unpaired t-test; Fig. 2), suggesting that both types of pitch patterns were similar attractors of prominence.

Subject-specific averages of prominence ratings for standard and falling F0 targets were then subjected to mixed-design ANOVA using stimulus type as the within-subjects factor and familiarization condition (RSC vs. FSC) as the between-subjects factor. Fig. 3 shows the average prominence ratings from the comparison. The analysis revealed no main effect of stimulus type $(p=$ $0.984)$ but a significant interaction of stimulus type and familiarization condition $(F(1,16)=6.331$, $p=0.023$, partial eta squared $\eta^{2}=2.84$ ). In other words, less frequent pitch trajectories (deviants) 
were considered as more prominent independently of the actual direction of F0 change during the word ( $M=1.094$ and $S D=0.529$ for deviants; $M=0.750$ and $\mathrm{SD}=0.274$ for standards).

Since the lexical content of the stimuli was fully independent of the F0 trajectory, each subject hearing exactly the same number of repetitions for each sentence, and each sentence occurring exactly the same number of times for both falling and rising pitch across both subject groups, we could also pool the responses using different combinations of the standard/deviant criterion and the absolute direction of the F0 change. Fig. 4 shows the perceived prominence levels across the possible pair-wise comparisons when all responses from all subjects are pooled together (Bonferroni-corrected for multiple comparisons). As already suggested by Fig. 3, a change of preferences is also observed in the pooled-responses-analysis (Fig. 4, bottom panel) where falling pitch is more prominent for RSC subjects habituated with frequent rising pitch $(M=1.100, S D=$ 0.750 for falling and $M=0.758, S D=0.620$ for rising; $Z=4.09, p<0.001, r=0.193$; Wilcoxon rank-sum test). In a similar manner, subjects habituated with predominantly falling pitch consider words with a rising pitch significantly more prominent $(M=1.089, S D=0.681$ for rising versus $M$ $=0.678, S D=0.742$ for falling; $Z=4.23, p<0.001, r=0.199)$. No differences in prominence ratings are observed when comparing rising deviants to falling deviants, rising standards to falling standards, or falling and rising stimuli in overall.

Subject reports from debriefing did not contain any systematic patterns related to the experimental design or stimulus naturalness.

\subsection{Discussion on experiment 1}

The results from experiment 1 support the initial hypothesis that prominence and unpredictability of the acoustic prosodic features are connected, as the impact of low probability F0 on subjective impression of word prominence is clearly seen in the data. Even though a rising F0 on the wordinitial syllable is typically used to signal accentuation, also in Finnish tested here (Suomi, Toivanen \& Ylitalo, 2008), a 5-minute exposure to F0 trajectories with a predominantly rising F0 was sufficient to negate the effect of rising F0 as a marker for sentence prominence. In contrast, predominantly falling F0 during habituation led to significantly larger ratings of prominence of words with rising F0. This suggests that the subjects in this group, despite being already biased towards using rising F0 as a cue for prominence, were also sensitive to the distributional properties of the prosodic trajectories during the task. In addition, statistical adaptation occurred even though the subjects were not asked or guided to focus on the intonation in any manner, but were given a semantic decision task instead. Since there were no lexical or semantic differences between the lowand high-predictability targets, the present results also show that this type of expectation-based prosodic processing occurs in parallel with lexical processing. However, the present experimental setup with a forced-choice prominence annotation task does not ensure that the listeners were actually treating prosodic deviants as linguistically meaningful patterns, especially since the stimuli were artificially generated by manipulating F0 contours of previously recorded utterances. This issue was addressed in a second experiment by using EEG measurements to probe real-time semantic and probabilistic processing of the stimuli, as described next. 


\section{Experiment 2: Electrophysiological markers of prominence after statistical exposure}

The first experiment revealed that a short-term exposure to prosodic patterns from a specific distribution can alter the subjective impression of word prominence. However, it is possible that the subjects considered deviant F0 trajectories as more salient because they were less frequent, but this does not dictate that the saliency played any linguistic function in the task. Instead, the subjects might simply have marked words with less likely F0 trajectory as prominent since they were asked to translate their perceptual auditory impressions into word-level prominence markings in a forcedchoice task (with a null option). It was also unclear how much the listeners were actually attending to the prosody of the stimuli during the habituation stage despite the semantic decision task they were asked to perform. For instance, the subjects might have started to consider prosody as a taskrelevant factor if they became consciously aware of the prosodic manipulation. Even though none of these factors were systematically reported during the debriefing of the subjects after the first experiment, such factors cannot be ruled out based on the first experimental design.

The second experiment was aimed to resolve these factors by measuring EEG-based ERPresponses from two groups of listeners when they were exposed to stimuli and task similar to the habituation stage of experiment 1 . We chose to use EEG since it provides a window to real-time processing of speech input, allowing us 1) to further verify that subjects indeed track probabilities at the suprasegmental level in continuous speech during a semantic decision task, 2) to see whether the probabilistic processing at the prosodic level interacts with the neural markers for semantic processing of incoming words with the same type of stimuli that were used for experiment 1, and 3) to see whether there are any neural indicators for subjects considering prosodic deviants as taskrelevant stimuli in their overt semantic decision task. The ERP components relevant to these three questions are briefly discussed below.

\subsection{Relevant EEG components and expected outcomes}

Our main interest was to quantify any observable differences in event-related potentials between the standard and deviant conditions across the subjects when the only differentiating factor between the responses would be the subjective probability of the F0 trajectory with all other linguistic and acoustic factors averaging out in the ERP responses. Based on the existing literature on ERPs, we were specifically interested in N200, P300, N400, and P600 components.

First of all, the current prosodic oddball-paradigm was expected to lead to an increased negative amplitude around 200-ms after F0 manipulation onset during the deviant conditions as long as the listeners are tracking relative probabilities of the prosodic trajectories despite being focused on lexical semantics. This so-called auditory N200 component, also known as the auditory mismatch negativity $(\mathrm{MMN})$, is typically observed at frontal scalp locations and does not require conscious attention to the stimuli (see, e.g., Näätänen, Paavilainen, Rinne \& Alho, 2007, or Garrido, Kilner, Stephan \& Friston, 2009, for reviews). Although early MMN studies were largely based on the oddball paradigm where an auditorily deviating stimulus occurs randomly between repeating standard stimuli, it is now known that MMN is also observed for statistical or structural violations in sequences of stimuli, including tones (Tervaniemi et al., 1994) and syllables (Francois et al., 
2017), lower probability sequences being associated with larger MMN amplitudes (Koelsch et al., 2016; see also Näätänen et al., 2007). In addition, MMN is associated with involuntary attentional shifts towards surprising auditory stimuli (e.g., Escera, Alho, Winkler \& Näätänen, 1998, and references therein). MMN-like ERP component is also observed after the onset of words that are incongruent in a given sentential context, and this has been hypothesized to correspond to a lexical selection process where lexical predictions and actual phonemic content interact (Connolly \& Phillips, 1994; van den Brink, Brown \& Hagoort, 2001). In the context of prosody, Tong et al. (2014) have previously shown that adult listeners have increased N200 amplitudes for words mother and today when they have a less frequent prosodic realization in comparison to a baseline prosody, revealing that listeners are tracking relative probabilities of suprasegmentals in isolated repeated word forms. For our current purposes, N200 serves as a replication target for the study of Tong et al. (2014), but now using continuous four-word utterances with higher lexical variability instead of using two isolated words repeating in a close temporal proximity. If the subjects are indeed tracking probabilities at a prosodic level when the lexical content is equally predictable in both conditions, they should exhibit larger N200 amplitudes for the deviant condition, independently of whether they are consciously attending to the prosody and independently of whether they are considering F0 manipulations as linguistically significant.

As another relevant component, auditory P300 is a slow positive wave at centro-parietal regions that peaks around 300-ms post-stimulus onset and is typically associated with task-relevant stimuli in a go/no-go oddball paradigm (see Polich, 2007, for an overview). P300 amplitude is largely driven by the temporal interval between the task targets, longer intervals leading to larger amplitudes, which translates to larger amplitudes for low-probability targets in commonly used experimental designs (Gonsalvez \& Polich, 2002). P300 is also typically large and robust and requires relatively few trials to measure in controlled conditions (e.g., Cohen \& Polich, 1997). P300 can be divided into P3a and P3b components where P3a can be elicited for auditory oddballs without an explicit task whereas P3b is only observed in the context of stimulus-relevant tasks (Polich, 2007). P3a typically peaks at $250 \mathrm{~ms}$ and is more frontal than P3b (Picton, 1992). Since P300 amplitude is inversely related to the probability of the stimulus, an increased P3b-like positivity for deviant stimuli would suggest that the subjects are considering prosodic manipulation as task-relevant in our present experiments.

In addition, P600, a positive component that peaks around $600 \mathrm{~ms}$ after stimulus onset at centro-parietal electrodes, is largely associated with syntactic parsing in the context of language input (e.g., Gouvea, Phillips, Kazanina \& Poeppel, 2010, and references therein). For instance, P600 is observed in the context of grammatical errors and garden-path sentences (e.g., Osterhout \& Holcomb, 1992). It has therefore been interpreted as a component that involves reanalysis of the preceding input to properly account for the present information (Kaan \& Swaab, 2003), or simply as a reflection of the added time required to build a coherent representation for more complex input (Hagoort, 2003). In the present study, presence of differences in P600 amplitude between conditions would suggest that the listeners undergo some type of re-interpretation or more extensive linguistic analysis of the utterance that depends on the probability of the observed prosodic pattern, as measured by recently experienced prosodic distributions. 
Potentially the most interesting ERP component in the present study is, however, N400. N400 is associated with semantic processing of perceptual input, its amplitude increasing for more unexpected or incongruent stimuli in the given context (Kutas \& Hillyard, 1980; Holcomb \& Neville, 1990). For instance, N400 amplitude is increased when subjects are visually shown with semantically unrelated object pairs (Barret and Rugg, 1990), when a word is not congruent with the preceding context (e.g., Van Petten et al., 1999, and references therein), and when words are shown with incongruent visual objects (Francois et al., 2017). Spreading of word-related semantic priming, a process indexed by N400, is also known to operate without conscious control (Kiefer, 2002). In general, in their comprehensive review on N400, Kutas and Federmeier (2011) summarize that “...N400 region of the ERP is more accurately described as reflecting the activity in a multimodal long-term memory system that is induced by a given stimulus during a delimited time window as meaning is dynamically constructed" (boldface added), (see also Laszlo and Federmeier, 2011).

In the context of prosody, Steinhauer, Alter and Friederici (1999) initially showed that prosody has an immediate impact on the syntactic parsing of the temporally evolving speech input. More specifically, when lexical and syntactic expectations primed by the preceding prosodic contours were violated, a larger N400 and a following P600 were observed in contrast to baseline congruent input, suggesting that lexico-syntactic and prosodic parsing of the input are in close interaction during speech perception. Following studies confirmed that the interaction and priming of expectations flow both ways. For instance, Hruska et al. (2001) showed that when syntactically primed focus words (new informative words given the preceding discourse context) were missing an expected accentuation, a large posterior negativity was observed in ERPs. However, superfluous accentuation did not lead to a similar effect (Hruska et al., 2001). In contrast, Magne et al. (2005) observed that centro-parietal N400 amplitude is larger for sentence-final words that have pragmatically incongruent accentuation given the preceding (lexical) discourse context independently of whether the accent is missing of superfluous. However, they also reported P300 instead of N400 for similar mismatches in sentence medial positions, making the picture much more complicated. A series of studies have since investigated the neural signatures for linguistically or visually primed prosodic expectations in an attempt to disentangle the relationship between different factors and various observed ERP components during contextually congruent and incongruent prosodic input (Eckstein \& Friederici, 2005; Toepel, Pannekamp \& Alter, 2007; Mietz, Toepel, Ischebeck \& Alter, 2008; Pannekamp, Meer \& Toepel, 2011; Bögels et al., 2011; Hruska et al., 2001; Dimitrova et al., 2012; Honbolygó et al., 2016). Some of these studies have suggested that listeners are more sensitive to (unexpectedly) missing accentuation on focus words than (unexpected) superfluous accentuation on non-focus words (e.g., Hruska et al., 2001; Bögels, 2011), leading to the so-called Missing Accent Hypothesis (Bögels et al., 2011). On the other hand, Dimitrova et al. (2012) have suggested that the resulting ERP signatures depend on whether the listeners are actively engaged in a task that involves evaluation of the well-formedness of the prosody, or whether surprising prosodic patterns take place during normal discourse where larger N400s are observed for unexpected accentuation of words outside linguistic focus (Dimitrova et al., 2012; see also Toepel \& Alter, 2004, for related findings).

Finally, accentuation and N400 have been associated with the depth of processing of the speech input. For instance, the study of Wang et al. (2011) suggested that accentuation of 
incongruent focused words increased their depth of processing as indicated by the elicitation of a large N400. In contrast, in conditions where focus and accent were mismatching or where accentuation was absent, smaller N400 effects were observed, leading the authors to conclude that context and accentuation influence the depth of the semantic processing (Wang et al., 2011). Similarly, Li and Ren (2012) found that accentuation can influence the depth of semantic processing during spoken language comprehension, showing that accented semantically incongruent words elicit a large N400 effect. However, lexical incongruences (within a predictive context) were only observed in N400 when the incongruent words were accented, even though lexical surprisal should normally elicit a larger N400 for incongruent words independently of prosodic considerations. Their findings also suggested that accentuation does not only impact spoken language comprehension through semantic interpretations, but also through top-down factors such as syntactic or semantic predictability that can modulate the temporal selective attention of the listener (Li \& Ren, 2012).

Despite the somewhat divergent findings, the previous studies clearly indicate that the listening context gives rise to certain prosodic expectations, and that violations to these expectations (e.g., a missing expected accent, a superfluous accent, or both) are typically associated with a number of ERP effects depending on the task demands and type of stimuli. In addition, it seems that accentuation of syntactically or semantically unpredictable words modulates the depth of processing of those words and is largely based on top-down expectations (Wang et al., 2011; Li \& Ren, 2012), potentially enabling re-evaluation of their role in the information structure of the sentence. This process is often observed as a larger N400 component due to the additional effort in constructing a coherent semantic interpretation of the input (see Dimitrova et al., 2012, and section 4 for discussion). This observation also fits to the picture of prosodic surprisal as a tool for the talker to emphasize any arbitrary part of the message at will, as discussed in the introduction.

However, according to our knowledge, all the studies investigating prosodic expectations have used language-typical prosodic outcomes as "standards" and contrasted them against atypical prosodic outcomes, thereby making use of listeners' life-long experience with their native language. In addition, only the study reported by Honbolygó et al. (2016) has investigated prosodic expectations independently of concurrent lexical constraints (but still using a contrast between language-typical and atypical prosody), showing that ERP markers for prosodic parsing, such as the closure positive shift (CPS) for intonational boundaries and P600 for syntactic re-analysis, are also observed for sentences consisting of nonsense pseudowords.

In the present experiment, it was of interest whether semantic processing, as indicated by $\mathrm{N} 400$, is modulated by predictability of prosody when there is no preceding lexical, semantic, or syntactic context that would be informative of the prosodic outcomes for the target words. If deviant prosodic trajectories would show a larger N400 independently of the absolute direction of F0 change, this would suggest that prominence perception is affected by rapid adaptation to the statistical properties of the input, that is, that the cues for prominence are also easily malleable during adulthood when faced with novel type of speech. In contrast, a lack of interaction between prosodic probabilities and N400 amplitude together with a presence of N200 would suggest that the prosodic probabilities are tracked by the participants, but unlike in previous studies with languagetypical prosodic patterns, not interpreted as linguistically relevant by the listeners. 


\subsection{Methods for experiment 2}

\subsubsection{Stimuli and experimental design}

The speech stimuli were otherwise the same as in habituation stage of experiment 1 , but we extended the original set of semantically coherent habituation sentences from 10 to 20 unique utterances to increase lexical diversity of the input due to the longer duration of the experiment needed for robust EEG measurements. Again, for each utterance, rising and falling F0 versions of the sentence-final word were created (see section 2.2 for stimuli preparation). Subjects were again randomly assigned into two counter-balanced groups (falling standard and rising standard) with the constraint that both groups had the same overall number of subjects.

In contrast to experiment 1 , there were no separate familiarization and testing stages, but the EEG was measured continuously starting from the onset of the familiarization stage. The stimuli were arranged into four counter-balanced blocks. Each block was generated by first creating 10 subblocks, each sub-block consisting of presentation of all the 20 unique target utterances with 2 of them being deviants and 18 being standards. Each sub-block had two different utterances as the deviants so that all utterances were used once as deviants in the overall set of 200 utterances in the block. Ten distractor utterances, sampled from the set of 6 unique distractors, were then interleaved within the stimuli using the same 1:9 F0 ratio, creating a total of 210 utterances per block (20 deviants, 180 standards, 10 distractors). Similar to the first experiment, the purpose of the distractor utterances was to act as targets for the overt task of detecting semantically incongruent sentences in the speech stream, and they were not included in the data analyses. The order of the sub-blocks and the stimuli within each sub-block were pseudo-randomized separately for each subject. A total of four blocks ( 4 x 210 utterances, 80 deviants, 720 standards) were measured for each subject, each block containing exactly the same stimuli but in different order. The overall duration of each block was approximately 12 minutes.

\subsubsection{Participants and procedure}

A total of eighteen $(N=18)$ native speakers of Finnish (average age $28.6 \pm 4.8$ years; 10 male) were recruited from the premises of Aalto University and University of Helsinki, Finland, and were rewarded with movie tickets for their participation in the experiment. All subjects were right handed, reported normal hearing and no history of neurological disorders. One of the subjects had also participated in experiment 1 one year earlier.

The subjects were tested individually in a sound-isolated and electrically shielded measurement room. They were asked to sit and position themselves comfortably and avoid excess movements during the experiment. The stimuli were delivered binaurally at $65 \mathrm{~dB}(\mathrm{~A})$ through two Genelec (8030A) loudspeakers located in front of the subject (approx. $\pm 45^{\circ}$ angle from the centerline, $1.5 \mathrm{~m}$ distance). They were instructed to listen carefully to each utterance being played through the loudspeakers, interpret the meaning of the utterance, and press a button on a keypad whenever they heard a semantically incongruent sentence. The subjects were given a break after every stimulus block in order to rest before continuing to the next block. 
The stimulus material, EEG recording process, and experimental setup were approved by the Ethics Review Board of Aalto University. All participants in the experiment gave written informed consent before participation.

\subsubsection{EEG data collection and pre-processing}

The EEG data were collected at the Aalto Behavioral Laboratory. All data were collected with a 32channel actiCAP (Brain Products GmbH, Munich, Germany), using $500 \mathrm{~Hz}$ sampling rate, and $\mathrm{FCz}$ as the reference electrode. After the data collection, all data were re-referenced to an average reference in order to treat all topographic regions and ERP-components of interest equally (Murray et al., 2008; Dien, 2017). All block-specific recordings were then downsampled to $100 \mathrm{~Hz}$ and filtered with a $1.3-40 \mathrm{~Hz}$ band-pass filter using an equiripple FIR design with 80 -dB stop-band attenuation, $-65-\mathrm{dB}$ ripple on the pass-band, and with $0.5 \mathrm{~Hz}$ transition bands, followed by a correction of the constant group delay. A relatively high $1.3-\mathrm{Hz}$ high-pass cutoff frequency was chosen to compensate for the lack of separate artifact removal that would cause problems to the counter-balanced experimental design, and this value was found to ensure that the measurements from the two conditions aligned within \pm 1 standard error of the mean (SE) before the onsets of the target words.

Data from two subjects were excluded from the analyses, one due to presence of excessive EEG artifacts that were present throughout the experiment (continuous blinking and biting) and one due to equipment failure. Directions of the standard/deviant F0 were adjusted accordingly for the measurement of the remaining subjects in order to maintain a fully counterbalanced experimental setup.

\subsubsection{Data analysis}

A three-way factorial design with multiple time windows and electrode positions for both trial conditions (standards and deviants) was used to analyze the ERPs of interest. Based on visual analysis of the waveforms and previous literature on N200, P300, N400, and P600, we used four overlapping time windows centered around the expected latency of each component of interest (150-300, 200-450, 300-550, and 500-800 ms from the onset of the target word) to capture potential differences in the four ERPs between the prosodic standards and deviants, averaging the EEG-responses across each window. Note that we did not use difference waves between the responses to standards and deviants (as is typically done for quantifying the MMN response), and therefore we refer here to a potential negative deflection at the 150-300 ms time window as N200 rather than MMN. As for the electrodes, eight regional electrode channels [F3, F4], [Fz], [FC1 FC2], [C3, C4], [Cz], [CP1, CP2], [P3, P4], and [Pz] were used to cover the relevant portion of the anterior-posterior axis, centered on the midline and with varying degrees of lateralization, assuming that the ERP-effects of interest are symmetric across the two hemispheres (Fig. 5). For the channels with two electrodes, the mean voltage across the electrodes was used in the analyses. Before averaging across trials, epoch DC-levels were normalized using 500-ms of stimulus-preceding signal as the baseline. 


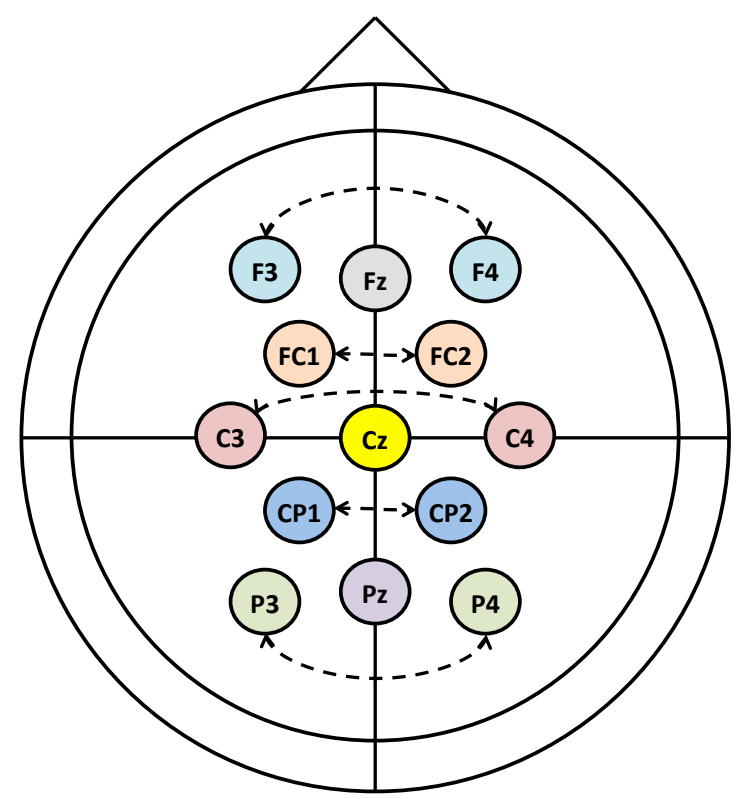

Figure 5: Electrodes and the eight EEG channels used in the data analysis. Channels are coded with colors and with the dashed lines connecting the electrodes belonging to the same channel.

Three-way repeated measures ANOVA (time window $\mathrm{x}$ electrode $\mathrm{x}$ condition) was first carried out for the subject mean responses to test if a main effect and any interactions between the experimental condition (standard vs. deviant F0) and the electrode positions and time windows existed. All repeated-measures statistics (incl. degrees of freedom) are reported after GreenhouseGeisser correction for sphericity using $p<0.05$ as the threshold for significance. In addition, only the potential effects related to the experimental condition are reported due to their interpretability. Any significant interactions were then disentangled by post-hoc paired t-tests between the two conditions using the subject mean responses for each combination of an electrode and a time window, and using Holm-Bonferroni correction for multiple comparisons.

\subsection{Results}

When first asked about general free-form feedback about the stimuli, almost all subjects commented about the contents of the sentences, indicating that they were focusing on the semantic disambiguation of the sentential content. Only two subjects commented on the naturalness of the stimuli, reporting that they sounded synthesized or unusual. None of the subjects reported having been aware of the probabilistic structure of the prosody or even mentioned prosody or intonation. When asked more specific questions about the intonation, many subjects explicitly noted that they did not notice any differences in the intonations as they were focusing on the semantic interpretation of the utterances. The latter also became evident through individual comments in which subjects described specific sentential contexts or full utterances that they believed to be incongruent. Many subjects commented that, when listening to the sentences, they were trying to determine for each sentence whether it was rational or not. Overall, this suggests that the subjects were not actively attending to the F0 trajectories during the listening task and that the F0 manipulations were not found to be distracting or distinct from other aspects of the speech signals.

Fig. 6 shows the grand average responses for all measured electrodes and across all $N=16$ subjects from both experimental groups. ANOVA revealed a significant main effect for condition 
$\left(F(1,15)=12.37, p<0.01, \eta_{\mathrm{p}}^{2}=0.45\right)$. In addition, there was a significant two-way interaction for time window $\mathrm{x}$ condition $\left(F(2.11,31.63)=4.49, p=0.018, \eta_{\mathrm{p}}{ }^{2}=0.23\right)$, and a three-way interaction for time window $\mathrm{x}$ electrode $\mathrm{x}$ condition $\left(F(2.22,33.25)=10.00, p<0.01, \eta_{\mathrm{p}}{ }^{2}=0.40\right)$. Since ANOVA revealed a main effect and interactions for condition, pair-wise post-hoc tests between conditions were carried out for all channels and time-windows and are reported in Table 1.
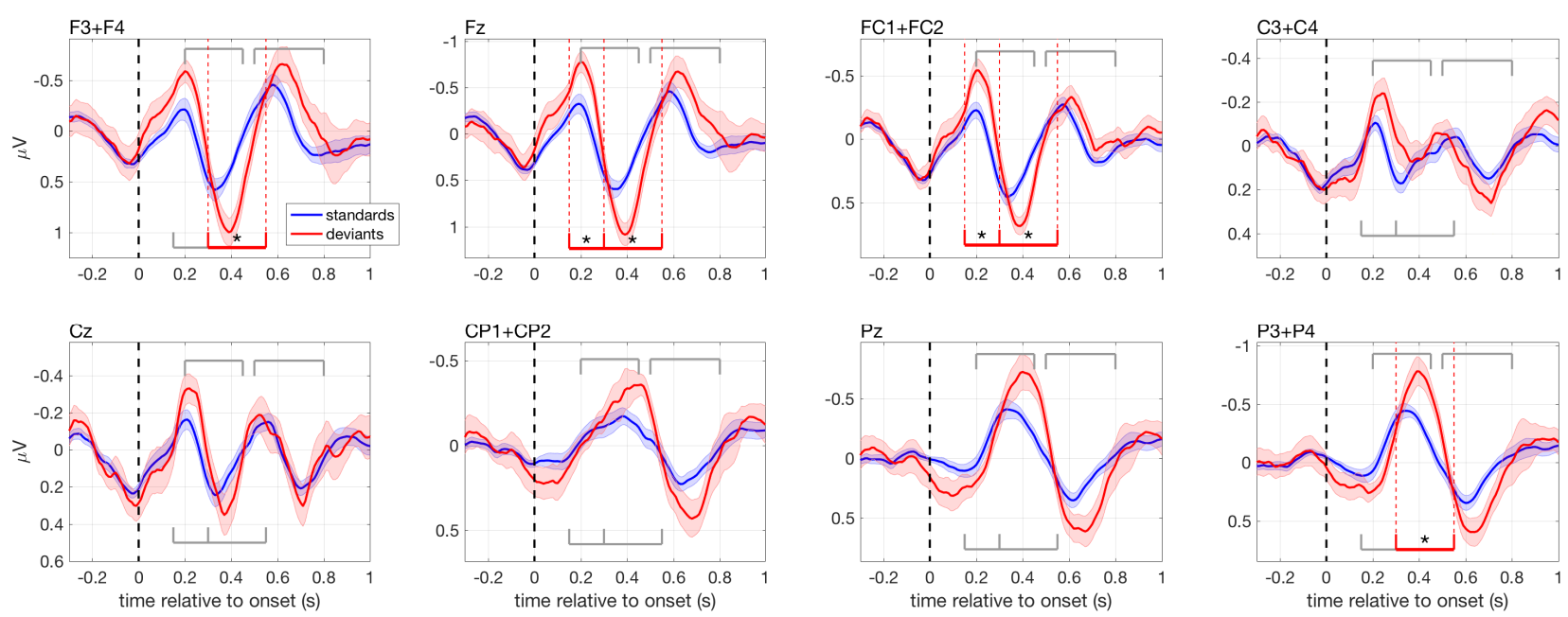

Figure 6: Grand average responses for all analyzed electrode channels in standard (blue) and deviant conditions (red). The curves are low-pass filtered with 100-ms moving average filter with delay correction for visualization purposes. Standard errors (SEs) of the averages across the 16 subjects are shown with shadowed areas. Time windows $(150-300,200-450,300-550$, or $500-800 \mathrm{~ms}$ after target word onset) with statistically significant differences are indicated with asterisks and red vertical bars (see also Table 1).

Table 1: Pair-wise post-hoc test results (paired t-test) between conditions for each time window and electrode channel using subject average responses for each condition. Adjusted p-values are reported with Holm-Bonferroni multiple-comparisons correction for $p<0.05$ and with $d f=15$ for all tests.

\begin{tabular}{|c|c|c|c|c|c|c|c|c|}
\hline channel & \multicolumn{2}{|c|}{$F 3+F 4$} & \multicolumn{2}{|c|}{$\mathrm{Fz}$} & \multicolumn{2}{|c|}{$\mathrm{FC} 1+\mathrm{FC2}$} & \multicolumn{2}{|c|}{$\mathrm{C} 3+\mathrm{C} 4$} \\
\hline time (ms) & $p$ & $t$ & $p$ & $t$ & $p$ & $t$ & $p$ & $t$ \\
\hline $150-300$ & 0.143 & 3.137 & $0.044 *$ & 3.826 & $0.001 *$ & 5.687 & 0.760 & 2.088 \\
\hline $200-450$ & 1.000 & 0.886 & 1.000 & 0.799 & 1.000 & 0.565 & 0.564 & 2.315 \\
\hline 300-550 & $0.044^{*}$ & 3.848 & $0.018^{*}$ & 4.318 & $0.011^{*}$ & 4.586 & 0.564 & 2.346 \\
\hline $500-800$ & 0.106 & 3.367 & 0.131 & 3.224 & 0.522 & 2.442 & 0.783 & 2.032 \\
\hline channel & \multicolumn{2}{|c|}{$\mathrm{Cz}$} & \multicolumn{2}{|c|}{$\mathrm{CP} 1+\mathrm{CP} 2$} & \multicolumn{2}{|c|}{$\mathrm{Pz}$} & \multicolumn{2}{|c|}{$\mathrm{P} 3+\mathrm{P} 4$} \\
\hline time (ms) & $p$ & $t$ & $p$ & $t$ & $p$ & $t$ & $p$ & $t$ \\
\hline $150-300$ & 0.522 & 2.434 & 1.000 & 0.327 & 1.000 & 1.381 & 1.000 & 1.603 \\
\hline $200-450$ & 1.000 & 0.923 & 1.000 & 1.562 & 1.000 & 1.353 & 1.000 & 1.044 \\
\hline 300-550 & 1.000 & 0.440 & 0.198 & 2.952 & 0.085 & 3.491 & $0.037^{*}$ & 3.953 \\
\hline $500-800$ & 1.000 & 0.535 & 0.629 & 2.224 & 0.131 & 3.218 & 0.121 & 3.283 \\
\hline
\end{tabular}

The first main finding visible in Fig. 6 and Table 1 is that the deviant condition exhibits a much larger negative peak centered at $200 \mathrm{~ms}$ than the standard condition. The peak is localized to the fronto-central electrodes $\mathrm{FC} 1+\mathrm{FC} 2$ and $\mathrm{Fz}$, corresponding well to the characteristics of typical auditory N200/MMN (see section 3.1).

The second main finding is that there is a large and slow wave peaking at approximately 400 ms, showing as negative in the parietal electrodes and as positive in the frontal electrodes due to the use of an average reference. The amplitude of this wave is significantly larger in the deviant 
condition from the standard condition in the time window of 300-550 ms for Fz, P3+P4 and F3+F4 channels. It was also separately verified that the same component was visible in the original signals with FCz reference electrode as a negative peak in frontal, central and parietal electrodes (not shown separately). Therefore we interpret the wave as the N400 component as its overall characteristics (amplitude, timing, and parietal negativity) match well to the characteristics of N400, and since the present speech comprehension task is expected to elicit a clear N400 in both experimental conditions even if the experimental manipulation would not have an impact on the amplitude (e.g., Kutas \& Federmeier, 2011).

In terms of time windows of $200-450 \mathrm{~ms}$ and $500-800 \mathrm{~ms}$ from target stimulus onset, no significant differences are observed. Although a P300-like deflection is visible in the central channels and a P600-like slow positive wave peaking at 600-700 ms region is visible in the parietal channels ( $\mathrm{P} 3+\mathrm{P} 4, \mathrm{Pz}$, and $\mathrm{CP}+\mathrm{CP} 2)$, the differences are not statistically significant between the conditions. We also attempted to adjust the window positioning as a post-hoc investigation, but did not find reliable distinctions between the conditions for windows centered around $300 \mathrm{~ms}$ or located after $500 \mathrm{~ms}$ from the manipulation onset.

We also compared the average responses between targets with rising and falling F0 by averaging the responses for the two types of trajectories over both conditions, and comparing the resulting responses for each time window and electrode similarly to the comparison in Table 1 . This was done to ensure that different F0 directions were not driving different ERP components. In addition, we were interested in whether the native-language bias to perceive rising F0 as prominent (e.g., Suomi et al., 2008) present in the results of experiment 1 would show up in the EEG responses.

Fig. 7 shows the grand average responses for all eight channels for the rising and falling stimulus types. Full statistics for the comparisons can be found in Appendix A. No significant differences were observed between the responses to the rising and falling F0 in the given timewindows (Holm-Bonferroni correction for multiple comparisons), and the amplitudes are generally smaller than those observed in the deviant condition independently of the direction (Fig. 6). The only main difference visible in Fig. 7 is the systematically shorter latency of the waveform during rising F0 in comparison to falling F0, although this was not separately quantified and does not show up in the comparisons using the pre-defined time windows. While the reason for the latency differences is currently unknown, it may be related to listeners' higher familiarity with rising F0 trajectories in natural speech and hence their more efficient processing (Krishnan, Gandour, Ananthakrishnan \& Vijayaraghavan, 2015).

In total, the comparison between stimuli with rising and falling F0 shows that the absolute direction of F0 does not correlate with the N200 and N400 amplitudes in the present experiment while the probability of the F0 trajectory has significant consequences to the amplitudes of both ERPs, lower probability F0 inducing larger ERP amplitudes. 

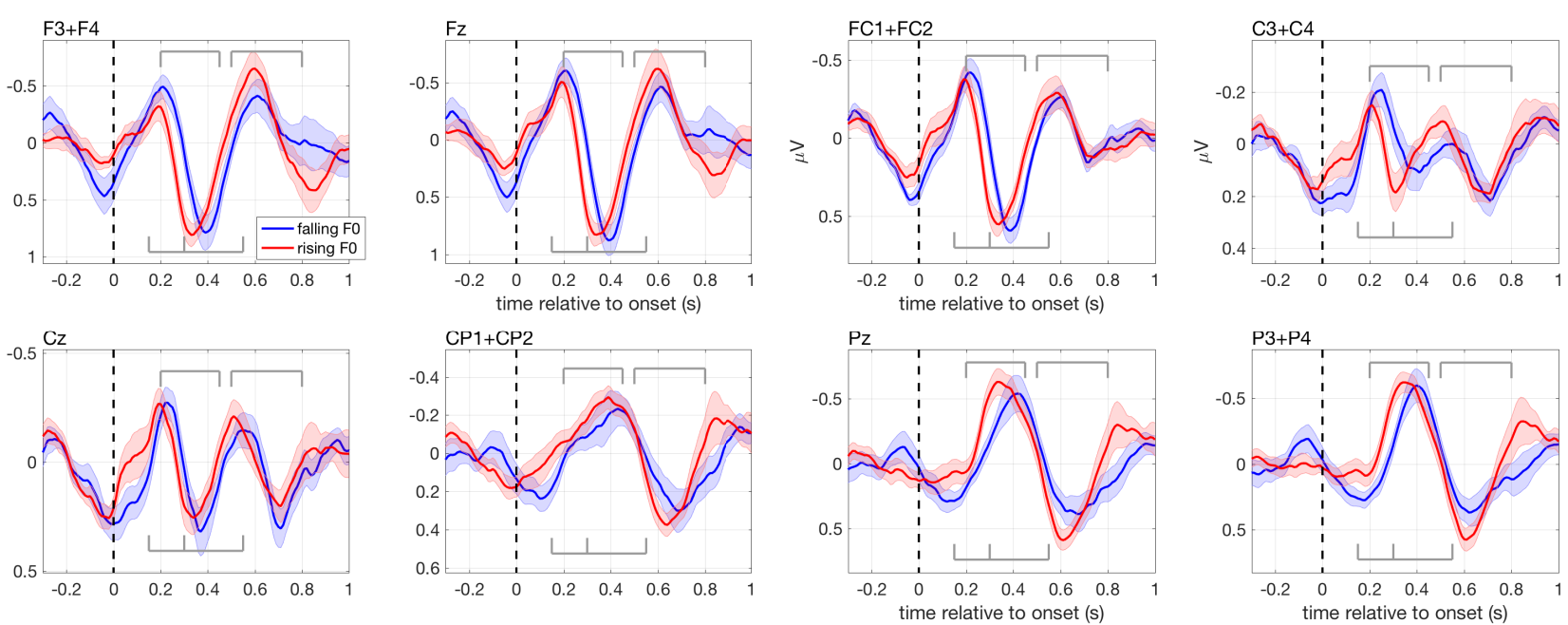

Figure 7: Grand average responses for all analyzed electrodes in rising (red) and falling (blue) F0 conditions. The curves are low-pass filtered with 100-ms moving average filter with delay correction for visualization. SEs are shown with shadowed areas. No statistically significant differences are present in the pre-defined time windows (150-300, 200-450, 300-550, or 500-800 ms after target word onset).

Finally, we also repeated the data analyses but now utilizing standard artifact removal by discarding data from all trials with channel waveform exceeding $\pm 100 \mu \mathrm{V}$, and also with a stricter $\pm 50 \mu \mathrm{V}$ criterion. No qualitative changes in the results were observed, as the findings in both cases revealed the same fronto-central N200 and parietal N400 effects (passing the significance criterion in the post-hoc tests; see Appendix B).

\subsubsection{Analysis of hemispheric differences}

Given that the combined channels resulted into robust N200 and N400 effects (Fig. 6, Table 1), we also investigated further whether there are, in fact, any observable differences between the responses from different hemispheres. This was done by repeating the main analyses described above, but now using a larger set of laterally positioned electrodes (midline electrodes were already covered in the above analyses). Only the electrodes from the occipital lobe and the most ventral electrodes on the temporal and frontal lobes were excluded since they either 1) had poor signal-tonoise ratio, likely due to problems in EEG-cap contacts at edges of the scalp (see also FP1 and FP2 in Fig. 8, for a concrete example), 2) are rarely reported to contain any of the ERP effects that are currently of interest (section 3.1). Fig. 8 shows the grand average responses from these electrodes and Table 2 describes the corresponding statistics. 

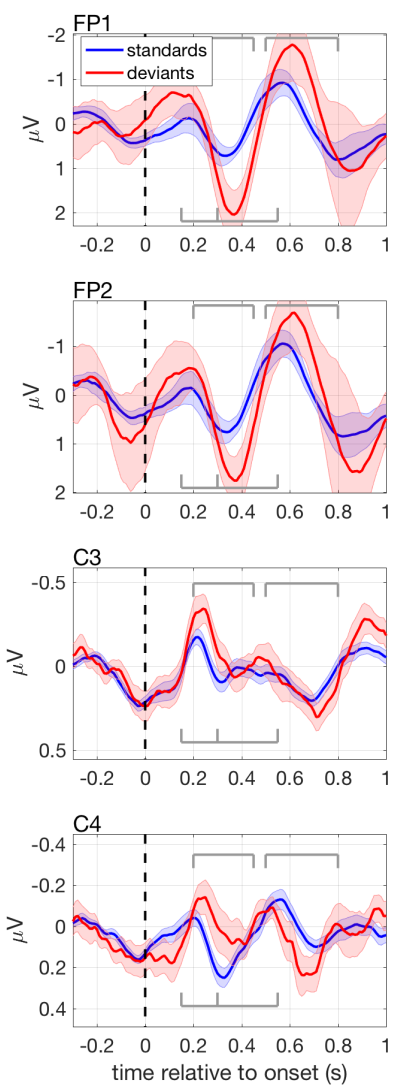
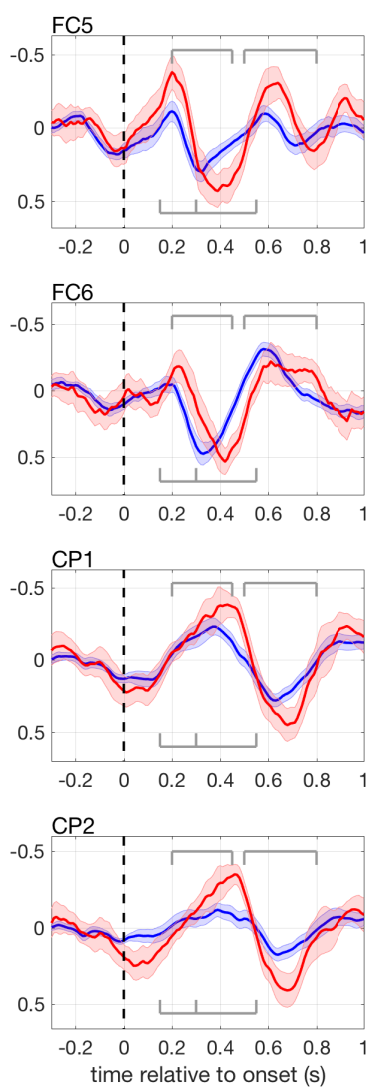
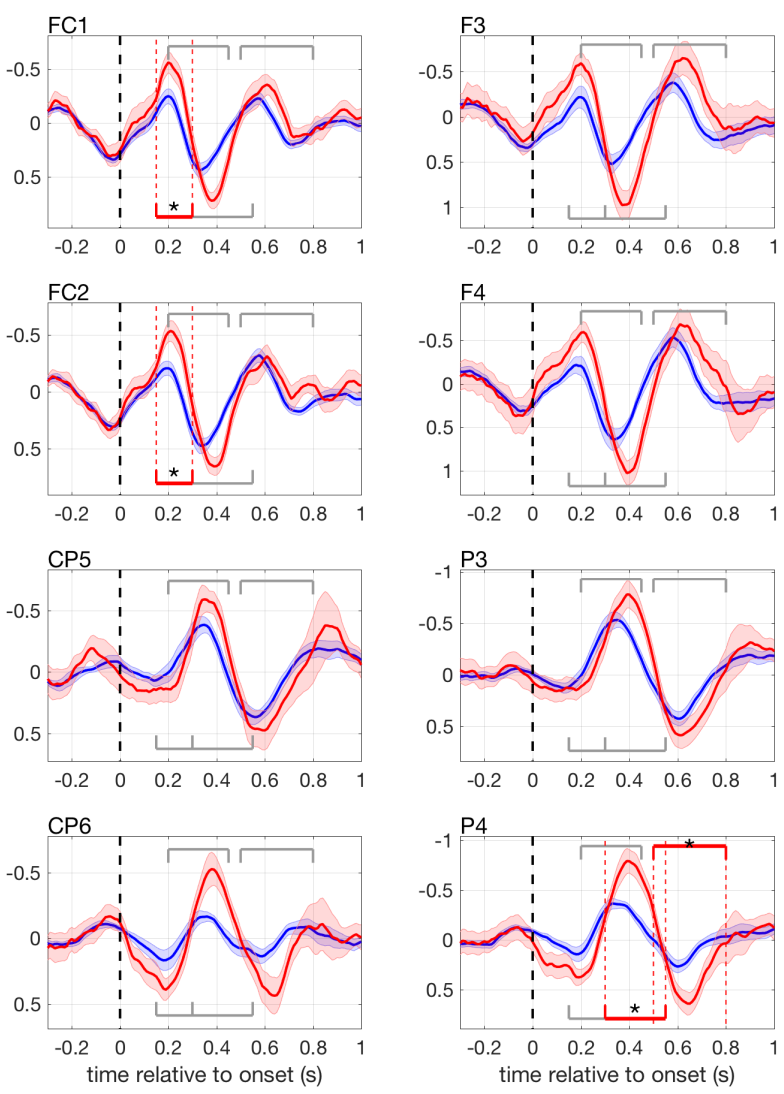

Figure 8: Grand average responses for all analyzed laterally positioned electrodes in standard (blue) and deviant conditions (red). The curves are low-pass filtered with 100-ms moving average filter with delay correction for visualization purposes. Standard errors (SEs) of the averages across the 16 subjects are shown with shadowed areas. Time windows (150-300, 200-450, 300-550, or 500-800 ms after target word onset) with statistically significant differences are indicated with asterisks and red vertical bars (see also Table 2).

Table 2: Pair-wise post-hoc test results (paired t-test) between conditions for each time window and laterally positioned electrode using subject average responses for each condition. Adjusted p-values are reported with Holm-Bonferroni multiple-comparisons correction for $p<0.05$ and with $d f=15$ for all tests.

\begin{tabular}{|c|c|c|c|c|c|c|c|c|c|c|c|c|c|c|c|c|c|}
\hline \multirow{3}{*}{$\begin{array}{l}\text { channel } \\
\text { time ( } m s)\end{array}$} & \multicolumn{8}{|c|}{$<-$ Anterior } & \multirow{2}{*}{\multicolumn{2}{|c|}{$\begin{array}{c}\text { Central } \\
\mathrm{C} 3 \\
\end{array}$}} & \multicolumn{6}{|c|}{ Posterior --> } & \\
\hline & \multicolumn{2}{|c|}{ FP1 } & \multicolumn{2}{|c|}{ F3 } & \multicolumn{2}{|c|}{ FC1 } & \multicolumn{2}{|c|}{ FC5 } & & & \multicolumn{2}{|c|}{ CP1 } & \multicolumn{2}{|c|}{ CP5 } & \multicolumn{2}{|c|}{ P3 } & \\
\hline & $p$ & $t$ & $p$ & $t$ & $p$ & $t$ & $p$ & $t$ & $p$ & $t$ & $p$ & $t$ & $p$ & $t$ & $p$ & $t$ & $\stackrel{9}{9}$ \\
\hline $150-300$ & 1.000 & 0.450 & 0.163 & 3.569 & $0.018^{*}$ & 4.690 & 0.652 & 2.806 & 1.000 & 2.222 & 1.000 & 0.090 & 1.000 & 0.859 & 1.000 & 0.971 & 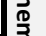 \\
\hline 200-450 & 1.000 & 2.000 & 1.000 & 1.521 & 1.000 & 0.215 & 1.000 & 0.111 & 1.000 & 2.066 & 1.000 & 1.309 & 1.000 & 0.984 & 1.000 & 0.625 & 范 \\
\hline $300-550$ & 0.768 & 2.693 & 0.146 & 3.630 & 0.052 & 4.140 & 0.855 & 2.605 & 1.000 & 1.509 & 0.729 & 2.740 & 0.740 & 2.722 & 0.257 & 3.315 & 需 \\
\hline $500-800$ & 1.000 & 2.489 & 0.316 & 3.200 & 1.000 & 2.424 & 1.000 & 2.187 & 1.000 & 1.008 & 1.000 & 1.631 & 0.965 & 2.533 & 1.000 & 2.240 & \\
\hline channel & \multicolumn{2}{|c|}{ FP2 } & \multicolumn{2}{|c|}{ F4 } & \multicolumn{2}{|c|}{ FC2 } & \multicolumn{2}{|c|}{ FC6 } & \multicolumn{2}{|c|}{ C4 } & \multicolumn{2}{|c|}{ CP2 } & \multicolumn{2}{|c|}{ CP6 } & \multicolumn{2}{|c|}{ P4 } & Dִ. \\
\hline time (ms) & $p$ & $t$ & $p$ & $t$ & $p$ & $t$ & $p$ & $t$ & $p$ & $t$ & $p$ & $t$ & $p$ & $t$ & $p$ & $t$ & 然 \\
\hline $150-300$ & 1.000 & 0.488 & 0.829 & 2.644 & $0.002^{*}$ & 5.810 & 1.000 & 1.385 & 1.000 & 1.372 & 1.000 & 0.752 & 1.000 & 1.840 & 1.000 & 2.321 & శ్ \\
\hline 200-450 & 1.000 & 2.282 & 1.000 & 0.138 & 1.000 & 1.015 & 1.000 & 1.105 & 1.000 & 2.164 & 1.000 & 1.680 & 1.000 & 1.867 & 1.000 & 1.614 & $\overline{0}$ \\
\hline $300-550$ & 1.000 & 1.953 & 0.213 & 3.429 & 0.255 & 3.332 & 1.000 & 1.358 & 1.000 & 1.849 & 0.504 & 2.953 & 0.257 & 3.319 & $0.038^{*}$ & 4.320 & ద్ర \\
\hline $500-800$ & 1.000 & 2.078 & 0.505 & 2.942 & 1.000 & 1.739 & 1.000 & 0.287 & 1.000 & 1.977 & 0.829 & 2.633 & 0.360 & 3.127 & $0.038^{*}$ & 4.320 & \\
\hline
\end{tabular}

As can be observed, both N200 and N400 are still significantly different between the two conditions, N200 occurring symmetrically in the fronto-central electrodes FC1 and FC2 whereas N400 now occurs only on the right hemisphere in parietal electrode P4. In addition, the P600-like deflection previously observed in the symmetrical analysis (Fig. 6) is now significant in the P4 electrode $(p=0.038, t=4.320, d f=15)$. 


\subsubsection{Effects of exposure time on N200, N400, and P600}

Finally, it was of interest how the observed ERP components (N200, N400, P600) evolved over the course of the second experiment. Fig. 9 shows the t-statistic from the paired t-tests between standard and deviant conditions as a function of time. The results are shown for the electrode channels FC1+FC2 for the time-window of 150-250 ms (N200) and for P3+P4 from 300-500 ms (N400) and 500-800 ms (P600). Time has been quantized so that responses from two subsequent experiment blocks are always averaged to obtain subject-specific mean responses for statistical testing, corresponding to 40 deviant and 360 standard trials per subject.

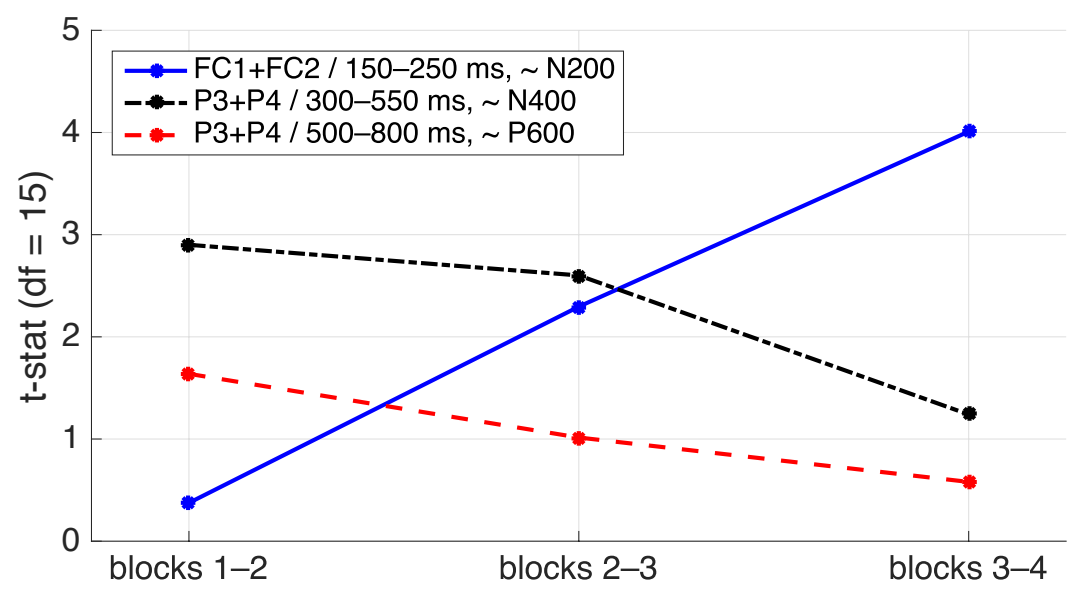

Figure 9: Evolution of the response differences between standard and deviant conditions for N200 (blue solid line), N400 (black dash-dotted line), and P600 (red dashed line) over the course of the experiment.

The temporal analysis shows that the time-course of N200 is very different from N400 and P600. More specifically, the effect-size of N200 increases as the subjects are exposed to more utterances while N400 and P600 are most prominent during the two first blocks and then decay towards the end of the experiment. This pattern of results is consistent with the notion that subjects reported increasing levels of fatigue towards the end of the experiment, which might have reduced attentiveness to the speech stream and thereby also linguistic processing of the stimuli. In addition, each possible combination of deviant prosody with a certain lexical item appeared once in every block, which might have reduced the size of N400 in later blocks as the same acoustic stimulus had already been encountered and processed earlier.

MMN (here measured as N200), on the other hand, is known to be a largely automatic response to unexpected stimulation and does not require active listening of the sound (Näätänen \& Winkler, 1999), and therefore it might also be more robust against subject fatigue. In addition, the amplitude of the MMN does not decay over time even if exactly the same deviant is encountered several times. Instead, the magnitude of MMN is known to increase over time for complex stimuli that require involvement of long-term memory in the acquisition of distributional properties of the stimuli (e.g., Winkler et al., 1996; Näätänen et al., 1997; see also Näätänen \& Winkler, 1999, for a review). In the current case, the stimuli are also structurally very complex: instead of having a typical MMN oddball-paradigm with repetitive stimuli occurring in brief succession, the probabilistic prosodic structure was embedded within the highly variable acoustic speech whose linguistic content was always uncorrelated with the trial type, and where the targets were always separated by several seconds of unrelated auditory stimulation. Therefore the observed differential 
response for standards and deviants requires that prosodic features are first separated from the segmental contents of speech, followed by some kind of probabilistic learning to capture the regularities at the prosodic level over temporal distances that are beyond the basic temporal window of auditory integration or echoic memory (Näätänen \& Winkler, 1999). Alternatively, the prosodic contours have to be maintained in some kind of stable representational form without interference from the lexical contents, causing this representation to change when the prosodic pattern is altered. In both cases, it seems unlikely that a simple short-term auditory buffer with a holistic representation of the full signal could carry out simple same/different-comparisons between the F0 contours of subsequent utterances, calling for more abstract processing of the input beyond straightforward low-level comparison of stimulus features.

The above reasoning would align with the predictive model of MMN by Garrido et al. (2009) who suggested that MMN observed at temporal cortices might correspond to mismatches between top-down predictions of low-level sensory input and the input itself, whereas failures of top-down predictions at more abstract representational levels are reflected by MMNs from the frontal sources (for more details, see Garrido et al., 2009, and references therein). However, accurate localization of the actual MMN generators is not possible given our current EEG setup. Also, even if the emergence of MMN between standards and deviants requires extensive learning due to the complex stimuli used, it is unclear why this is not the case for N400 whose grand average amplitude also only depends on the probabilities of prosodic trajectories irrespectively of the direction of F0 change or underlying lexical contents. Perhaps the semantic neurophysiological mechanisms underlying the N400 have more direct access to prosodic features extracted from speech due to existing excessive pre-test experience of the listeners in mapping linguistic stimuli into their meanings. In contrast, the N200 may depend on mechanisms that are shared and competed by many more factors such as domain general sensory and motor predictions at various levels of abstraction and hence requires sufficient repetition for prosodic contours to emerge as a dominant aspect in the temporal predictions. Alternatively, the mechanism behind N200 might be incapable of separating the lexical and suprasegmental acoustic features from each other, and hence N200 emerges slowly because it is conditioned on specific combinations of lexical items and F0 contours instead of F0 alone. However, resolving this issue is beyond the scope of the present study and is left for future work.

\subsection{Discussion on experiment 2}

The results from the EEG measurements reveal that the subjects are sensitive to the relative probabilities of different F0 trajectories even when they are not actively asked to pay attention to prosody but to focus on the semantic congruency of the utterances. In general, none of the discovered differences between standard and deviant conditions can be attributed to any physical or linguistic property of the stimuli, since the averages for both conditions are based on exactly the same set of speech signals played equal number of times, the only difference being the subjective probabilities of different $\mathrm{F} 0$ variants for each individual subject.

The presence of a significantly larger N200 component for deviant conditions confirms that the subjects are sensitive to the relative probabilities in suprasegmental acoustic features and that less likely prosodic outcomes elicit an N200 response, even when the deviants are acoustically 
convolved with the other linguistic content of the stimuli. This is in line with the finding of Tong et al. (2014) who found an MMN (N200) response for isolated words with different prosodic characteristics occurring at different probabilities. However, the present experiment shows that the tracking of probabilities also occurs in continuous speech with much richer lexical content than that used by Tong et al. (2014), and without temporally proximal repetition of the target words. This is noteworthy since the prosodic structure is inherently embedded in the same acoustic stream with the phonemic and lexical content of speech, and therefore our results indicate that listeners track acoustic probabilities (or are at least sensitive to rare / frequent distinctions) at multiple levels of auditory representation without specific task demands on the prosodic level. According to the prosodic predictability hypothesis presented by Kakouros and Räsänen (2016a), prosodic surprisal, prominence, and stimulus-driven attention are all aspects of the same mechanism for allocating more processing resources to specific aspects of the speech signal. The presence of prosody-driven MMN also aligns with this idea as frontal components of MMN have been previously associated with involuntary attentional shifts due to auditory changes (see Näätänen et al., 2007, for a review).

In terms of speech perception, the probability of the F0 trajectory was also found to modulate the N400 component of the neural responses to speech, deviant trajectories leading to larger N400 amplitudes. The mere presence of auditory MMNs for deviants would not have implied that the probability tracking of F0 trajectories is somehow connected to the analysis of the meaning in incoming speech, but could have been a result of a purely language-independent parallel processing of certain features in the auditory input. However, since N400 only occurs in tasks that tap to semantic processing, less frequent or contextually incongruent stimuli leading to larger N400 amplitudes (Kutas \& Federmeier, 2011), a negative correlation between F0 predictability and N400 magnitude in the present results suggests that surprisal at a prosodic level leads to altered semantic processing of the incoming speech. In addition, closer analysis revealed that the N400 was largely lateralized on the right hemisphere, aligning with the previous reports (see, e.g., Kutas \& Van Petten, 1994, for a discussion; see also Kutas \& Federmeier, 2011).

The present findings on N400 are also consistent with a number of previous reports on the influence of prosody on electrophysiological measures of speech perception. For instance, Magne et al. (2005) found that violation of expected presence or absence of accentuation led to a large increase in centro-parietal electrode negativity for sentence-final words whereas Dimitrova et al. (2012) found N400 for unexpected accentuation of non-focused words in a discourse context (see also section 3.1). The presently observed parietal responses are also remarkably similar to the N400-P600 pattern reported by Steinhauer et al. (1999) in the context of a mismatch between prosodically primed syntactic expectations in speech. The current results go beyond the existing studies by showing that the prosodic deviants influence N400 even when the prosodic expectations are not formed by the preceding syntactic or lexical context, but when the probabilistic structure is purely at the suprasegmental level and is independent of the information structure at the lexical level. In addition, the present results show that even a brief exposure to a certain distribution of prosodic patterns can lead to similar effects on N400 than the violation of language-dependent expectations investigated in earlier studies (e.g., Steinhauer et al., 1999; Magne et al., 2005; Wang et al., 2011; Dimitrova et al., 2012). 
What does increased N400 amplitude for prosodic violations during speech comprehension mean from a functional point of view? Friederici (1999) and Steinhauer et al. (2002) have interpreted increased $\mathrm{N} 400$ as a lexical re-access needed to account for syntactic anomalies observed during the first decoding pass of the incoming speech. However, it is unclear how the outcomes of this "second pass" would differ from the first one unless the second analysis somehow utilizes a broader set of alternative interpretations for the input. Since basically all documented factors contributing to the N400 amplitude are expectation-based, either primed through base frequencies or context-driven constraints such as word cloze probabilities (Kutas \& Federmeier, 2011), the size of N400 can be more generally viewed as a reflection of the neural activity ("effort") needed to settle to a new internally coherent representational state and the associated set of new predictions, given the preceding state and predictions of the brain. In this context, a large N400 would signal a conflict caused by input that does not conform to the expected stimulus outcomes. For instance, Van Berkum (2009) has proposed that N400 is an indication of "...the additional effort invested in semantic memory retrieval presumably involves the retrieval of a richer set of semantic features and associations for the semantic memory representation of the word at hand", i.e., of broader semantic processing beyond the largely top-down-dominated baseline parse when the input is highly predictable - an economical strategy for the predictive brain in largely redundant environments (cf., e.g., Federmeier, 2007; Friston \& Kiebel, 2009; see also introduction).

In the context of speech, the previous studies and current results suggest that the segmental and suprasegmental levels are jointly contributing to the prediction of the unfolding speech input at both levels. When these expectations are violated due to, e.g., incongruent syntax given the prosody (e.g., Steinhauer et al., 1999) or unlikely prosodic form given the syntactic construct (e.g., Magne et al., 2005) or expected prosodic outcomes in general (this work), the above type of more extensive semantic search is triggered in order to settle to a stable neural representation coherent with the input, as reflected by larger N400 amplitudes. What this means from a speech production point of view is that the talker can control listener's processing of the input without changing the lexical or syntactic properties of the message by tapping to the predictive mechanisms of the listener's (and talker's) brain, effectively modulating the information structure of the input with prosodic cues. As a result, prosodic accentuation and emphasis can be used to trigger deeper processing (Wang et al., 2011; Li \& Ren, 2012) or creation of alternative schemas in contrastive emphasis (e.g., "No, I walked home", "No, I walked home", "No, I walked home", or "No, I walked home") by introducing highly informative (low probability) events in an otherwise predictable perceptual stream, forcing the listener's brain to evaluate the input more thoroughly instead of allowing it to stick to the most likely interpretation given its current predictive state. In this context, N400 could be viewed as a reflection of the information value $-\log (\mathrm{p}($ stimulus $\mid$ context $))$ of the stimulus, where probability of the input is evaluated by the current context-dependent state of the listener's brain.

An alternative interpretation is that the increased N400 amplitude for prosodic deviants simply reflects additional processing cost for atypical input without any side-effects such as altered semantic processing or surprisal-enhanced learning. However, a major increase in ERP amplitude necessitates that there is a large number of additional spatially aligned neurons that are synchronously activated. It is difficult to picture such a large wave of synchronized activation without it resulting in any functional consequences as to how the input is interpreted or memorized 
or how future speech inputs of the same type are processed by the system, that is, without inducing any synaptic changes or residual priming of the semantic networks as a result. Still, this is a valid possibility and cannot be resolved by the present study.

In any case, the presence of an increased N400 amplitude for deviant intonation shows that prosodic parsing is inherently integral to the semantic decoding of speech (see also Steinhauer et al., 1999; Magne et al., 2005; Eckstein \& Friederici, 2005; Toepel et al., 2007; Mietz et al., 2008; Pannekamp et al., 2011), and that this is also true for languages without suprasegmental F0dependent phonemic contrasts, in this case, Finnish. Moreover, the mechanisms for this type of prosodic re-evaluation seem to be malleable based on short-term speech exposure even for subjects with tens of years of exposure to prosodic patterns from certain language-dependent distribution.

As for P300, no significant differences between the conditions were observed in the 200450 ms time-window typical to the P3b component (see section 3.1). Since previous research shows that P300 is usually observed in the context of task-relevant oddball stimuli (Picton, 1992) with oddball stimulus intervals increasing P300 amplitudes (Gonsalvez \& Polich, 2002), the result suggests that the prosodic oddballs may not have been considered as task-relevant modifications by the subjects, leading to task- and attention-independent N200 for deviants but not for P300. Other possibility is that any condition-specific effects in P300 were simply masked by the large amplitude modulation of the partially overlapping N400 component. Potential condition-dependent effects on P300 may become distinguishable with some other electrode reference and/or some type of sourceseparation technique applied to the measurements. However, our present data analysis procedure does not permit reliable verification of such findings.

Finally, our initial combined-channel analysis did not reveal any condition-dependent effects on P600, a neural marker associated with syntactic re-analysis of language input (section 3.1). However, the closer analysis with individual electrodes revealed a significant difference in P600 amplitude between the standard and deviant conditions in the right parietal lobe electrode (P3). Together with the preceding N400 observed in the same electrode, the overall shape of this biphasic deflection is remarkably similar to that reported by Steinhauer et al. (1999) who found a significant N400 and P600 modulation for incongruent syntax given the expectations arising from a prosodic context. This suggests that the prosodic deviants may also cause syntactic re-analysis of the message-a process with which P600 is typically associated (section 3.1), even if the syntactic structures of the sentence are equal in the compared conditions (see also Honbolygó et al., 2016). We find the evidence for both altered semantic processing (N400) and syntactic re-evaluation (P600) for prosodic deviants especially curious, considering the fact that none of the spontaneous listener reports contained any notion of prosody or its relation to the given listening tasks and since condition effects on P300 were not observable in the results. It seems that the potential effects of prosodic deviants do not necessarily penetrate consciousness when the listeners are focused on a semantic processing task, even when the depth or extent of processing is clearly different from the baseline prosodic forms (cf., Wang et al., 2011; Li \& Ren, 2012). However, our current experimental data cannot be used to address the issue of subject awareness in a systematic manner. 


\section{General discussion}

The present results show that listeners' subjective impression of prominence is dependent on the probability of the prosodic trajectory given a preceding prosodic exposure, and that this effect is observed independently of the actual direction of F0 change. More specifically, the first experiment shows that the listeners are more likely to mark sentence-final words as prominent when the F0 contour during the word is less frequent than the baseline F0 pattern during preceding exposure to similar speech. However, this finding could also be attributed to idiosyncratic listening strategies where the subjects might have simply translated prominence annotation task into an oddball detection task, marking atypical prosodic outcomes as prominent, and doing this independently of whether the F0 manipulations actually played any role in the real-time speech comprehension. The second experiment was designed to address this issue by probing real-time speech perception with electrophysiological recordings. The EEG-results show that words with less likely F0, given the preceding exposure, lead to significantly larger amplitudes in the N400 ERP component associated with altered semantic processing of the input, again independently of the actual direction of F0 change. In addition, some evidence for P600 (syntactic re-analysis) was also observed in post-hoc investigations. These findings indicate that surprisal caused by expectation violation at the acoustic prosodic level interacts with the concurrent decoding of word meanings independently of the preceding lexical or syntactic context, and suggests that the synthetic F0 contours were treated as linguistically valid cues during speech perception. In general, the findings support the prosodic predictability hypothesis (e.g., Kakouros \& Räsänen, 2016a) and are in line with the earlier findings where expectations primed by various linguistic factors have an impact on prosodic parsing of speech, or where prosodic context modulates parsing of the syntactic content of speech (e.g., Steinhauer et al., 1999; Magne et al., 2005; Eckstein \& Friederici, 2005; Toepel et al., 2007; Mietz et al., 2008; Pannekamp et al., 2011; Buxó-Lugo \& Watson, 2016; see also Bishop, 2012, for a discussion).

Perception of prosody is typically treated as the skill of knowing how the listener's language utilizes non-phonemic articulatory gestures and control over the voice source to convey prosodic structure. The present study shows that at least one aspect of prosodic structure, the differential impression of prominence on different words, although definitely influenced by the native-language factors, is also a dynamic process where recent speech experience plays a significant role and can even override typical cues for signaling prominence in the language. This is sensible from an ecological point of view, when the entire perceptual machinery is considered, as continuous tracking of regularities in recent sensory input enables adaptation to changing environmental circumstances, or in the case of speech, to changing speaker and language characteristics that may be difficult to generalize into one global model of how different linguistic and prosodic entities should invariantly sound (see also Kleinschmidt \& Jaeger, 2015).

In terms of linguistic theory, it does not necessarily make a big difference whether it is certain acoustic feature configurations as such that convey prominence or whether it is the predictability of these features that signals it. For a constrained production system such as the speech apparatus paired with the need to maintain intended phonemic contrasts, the creation of prosodic surprisal will necessarily boil down to the usage of a limited set of acoustic cues (F0, 
energy, tilt, duration) that show up systematic and measurable physical values in similar conditions and averaged across several tokens as documented by the large body of literature (Fry, 1955, 1958; Lieberman, 1960; Terken, 1991; Kochanski, Grabe, Coleman, \& Rosner, 2005; Sluijter \& van Heuven, 1996). However, in terms of understanding speech perception and learning, the predictability-based account is more parsimonious. This is because, in this case, the concept of prominence becomes an integral part of domain-general core cognitive mechanisms for (statistical) learning, the resulting top-down predictions in perception, and the expectation-based attentional mechanisms that allocate cognitive resources to the aspects of the sensory experience that are most informative under the current statistical model of the environment, thereby phenomenologically highlighting these aspects of the input over others. Most importantly, this type of predictive mechanism can learn and operate in a fully unsupervised manner and automatically adapts to changing circumstances or language environments. In contrast, by just stating that prominence is signaled by behavior $\mathrm{X}$ of feature $\mathrm{Y}$ calls for at least two additional explanations: 1) what is the cognitive function of this "prominence" and how does it interact with other perceptual processes, and 2) how do the listeners learn, in the absence of explicit feedback, that value $\mathrm{X}$ of feature $\mathrm{Y}$ signals prominence but some other equally likely combinations do not. Unless, of course, the perceptual system considers typical values of $\mathrm{Y}$ as non-prominent since they do not stand out, already transforming the formulation of the process into a probabilistic one.

A predictability-based account for sentence prominence may also help to understand infantdirected speech. It has been hypothesized that one function of IDS is to control attention of the child towards speech input (e.g., Soderstrom, 2007), and it is known that attention and statistical learning performance are connected (see Bosseler, Teinonen, Tervaniemi \& Huotilainen, 2016, for a recent overview). However, at the same time, children's capability to utilize prosodic cues in a linguistically meaningful manner seems to require years of learning (e.g., Cutler \& Swinney, 1987, and references therein), posing the question of how infants can still utilize prosodic cues of IDS without understanding their linguistic function. Since the basic auditory perception of gross prosodic contours (e.g., F0, energy, durational proportions) does not require any language-specific linguistic capabilities, regularities at the prosodic level may be readily available to a pre-linguistic infant during early word learning even if their role in the language is not known yet. As long as the infants are sensitive to such structure similarly to adults, the exaggerated intonation of IDS may have statistical properties that are initially easier to capture than the actual segmental level of speech, yet still varying enough to induce predictive uncertainty and hence enhanced attentional attraction towards the speech stream (cf. the Goldilocks-effect discussed in the introduction; Kidd et al., 2012; see Räsänen, Kakouros \& Soderstrom, 2017, submitted, for initial evidence). In addition, temporal variation in prosodic predictability may function as a "flashlight of information selection", thereby helping the caregiver and the child to coordinate in disambiguating relevant auditory information such as referential content words from the background and facilitating word learning when syntactic cues to the information structure of the utterances are not yet available for the child. However, the connections between exaggerated prosodic contours, their predictability at different points in time, and infants' attention to speech are currently largely unclear and should be investigated in the future. Note that if the predictive framework for prosody-driven attention is correct, learning of statistical regularities at the phonemic and lexical levels play a similar role in 
parallel to prosody by drawing attention to syllables and words that are surprising in an otherwise familiar context. The difference is that prosodic regularities such as intonation contours are initially much more perceptually accessible than the much more variable lexical and phonemic units, making it easier to create a contrast between the expected and unexpected (cf., Kidd et al., 2012).

Overall, a learning mechanism that tries to minimize future prediction error by specifically focusing on low-probability events in the auditory stream would provide an ecologically valid characterization for the filters present in the classical models of attention (Broadbent, 1958; Treisman, 1964), maximizing information flow to the stages following the attentional selection (cf., Shannon, 1948) without requiring hard-wired and a priori specifications for the filter characteristics. In the context of spoken language, suprasegmental non-phonemic cues can be used to pass this filter while keeping the linguistic content of the message fixed.

Another relevant aspect of the present results concerns the time-scale of the statistical adaptation. In experiment 1, exposure to five minutes of speech from a specific prosodic distribution was sufficient to alter the perception of prominence in contrast to native-language baseline while in experiment 2 the effects of statistical adaptation became visible in N400 already during the first two blocks of the experiments $(2 \times 12 \mathrm{~min})$ where exposure and measurement of the responses took place concurrently. Even though the overall number of deviant trials for each subject was not large even across all the two blocks $(N=40)$ and some number of trials was necessary for the subjects to learn the relative probabilities of the rising and falling F0, the difference in N400 ERP responses was clearly observable with only 16 test subjects. N200 effects, on the other hand, emerged more slowly and were most prominent during the last two blocks of the experiment. Although the current data do not allow the precise estimation of minimum exposure needed for a measurable impact on the prominence judgments, the result shows that the adaptation takes place at a much faster rate than what would be expected if the listeners weighted equally all speech "samples" across their personal history in the generation of the on-line prosodic expectations. This is also important to keep in mind when conducting experiments on prominence perception, especially prolonged EEG-measurements with many repetitions, as the testing procedure itself may reduce the apparent efficacy of some cues in signaling prominence or even introduce new ones.

As for the implications of such a short-term adaptation process, a bias on recent input can be viewed as functionally useful and also as expected from the point of view of statistical learning theories. This is because rapid recalibration of the attentional system to the structure of the present environment automatically makes sure that sensing and learning are always focused on aspects of the current environment that are not correctly predicted by the existing model, that is, inputs that carry information to the listener (cf., Shannon, 1948). In the context of speech, this could simply mean that rapid prosodic adaptation allows the listener to better cope with individual differences in speaking styles, voices, dialects, and accents that are notoriously difficult to capture and characterize with a static set of acoustic parameters, but where cues for relative information value of different sentence components are still likely to be present for each situation.

\subsection{Conclusions}

The present study provides further evidence that predictability of prosody is connected to the impression of prominence in speech, and that the prosodic predictability also interacts with the 
semantic processing of speech input. Moreover, expectations for the prosodic outcomes seem to depend on a recent exposure to speech input, as a brief exposure to speech with a specific distribution of F0 probabilities is sufficient to induce measurable changes in the acoustic cues for prominent words for listeners who have tens of years of experience in listening to their native language's prosodic patterns, indicating that the relationship between predictability and prominence is not just coincidental. In addition, the prosodic adaptation does not seem to require conscious attention to the prosodic aspects of speech, implying that tracking of suprasegmental regularities takes place automatically and implicitly during speech perception.

However, more work is needed to confirm the present findings under various circumstances and experimental manipulations, and to further explore the roles of probabilistic phenomena and statistical learning in the perception of suprasegmental aspects of speech. Beyond replication of the present findings in another settings and languages, it would be beneficial to conduct experiments using more natural stimuli and richer variability of prosodic contours and probability distributions, having target prosodic manipulations occurring at different sentential contexts and, e.g., manipulating the duration of pre-test exposure in prominence perception in order to get a handle on the various factors contributing to the emergence of prosodic expectations.

\section{Acknowledgements}

An initial report on the behavioral results of the first experiment was presented in the Annual Conference of the Cognitive Science Society held at Philadelphia, PA, in August 2016. Author S.K. was funded by Academy of Finland projects 312490 and 269279, O.R. by Academy of Finland projects 274479 and 312105, and N.S. was funded by Academy of Finland project 296751. The authors would like to thank Veli-Matti Saarinen for his assistance in conducting the EEG measurements.

\section{References}

Altosaar, T., ten Bosch, L., Aimetti, G., Koniaris, C., Demuynck, K., \& van den Heuvel, H. (2010). A Speech Corpus for Modeling Language Acquisition: CAREGIVER. Proceedings of the International Conference on Language Resources and Evaluation (LREC-2010), Valletta, Malta (pp. 1062-1068).

Aslin, R. N., \& Newport, E. L. (2012). Statistical learning: From acquiring specific items to forming general rules. Current Directions in Psychological Science, 21, 170-176.

Aylett, M., \& Turk, A. (2004). The smooth signal redundancy hypothesis: a functional explanation for relationships between redundancy, prosodic prominence, and duration in spontaneous speech. Language and Speech, 47, 31-56. 
Aylett, M., \& Turk, A. (2006). Language redundancy predicts syllabic duration and the spectral characteristics of vocalic syllable nuclei. Journal of the Acoustical Society of America, 119, 3048-3058.

Barret, S. E., \& Rugg, M. D. (1990). Event-related potentials and the semantic matching of pictures. Brain and Cognition, 14, 201-212.

Bendixen, A., SanMiguel, I., \& Schröger, E. (2012). Early electrophysiological indicators for predictive processing in audition: a review. International Journal of Psychophysiology, 83(2), $120-131$.

Bishop, J. (2012). Information structural expectations in the perception of prosodic prominence. In G. Elordieta \& P. Prieto (Eds.), Prosody and meaning (pp. 239-270). Berlin: Walter de Gruyter.

Bock, J. K., \& Mazzella, J. R. (1983). Intonational marking of given and new information: Some consequences for comprehension. Memory \& Cognition, 11, 64-76.

Boersma, P., \& Weenink, D. (2012). Praat: doing phonetics by computer [Computer program]. Version 5.3.13, retrieved from http://www.praat.org/

Bolinger, D. (1972). Accent is predictable (if you're a mind-reader). Language, 48, 633-644.

Bolinger, D. (1983). Intonation and gesture. American Speech, 59, 356-374.

Bosseler, A. N., Teinonen, T., Tervaniemi, M., \& Huotilainen, M. (2016). Infant directed speech enhances statistical learning in newborn infants: An ERP study. PLOS ONE, DOI:10.1371/journal.pone.0162177, September 12.

Buxó-Lugo, A., \& Watson, D. G. (2016). Evidence for the influence of syntax on prosodic parsing. Journal of Memory and Language, 90, 1-13.

Bögels, S., Schriefers, H., Vonk, W., \& Chwilla, D. J. (2011). Pitch accents in context: How listeners process accentuation in referential communication. Neuropsychologia, 49, 2022-2036.

Broadbent, D. (1958). Perception and communication. London: Pergamon Press.

Calhoun, S. (2010). The centrality of metrical structure in signaling information structure: A probabilistic perspective. Language, 86, 1-42. 
Campbell, N. (1995). Loudness, spectral tilt, and perceived prominence in dialogues. Proceedings of the 13th International Congress of Phonetic Sciences (ICPhS-1995), Stockholm, Sweden (pp. 676-679).

Campbell, N., \& Beckman, M. E. (1997). Stress, prominence, and spectral tilt. In A. Botinis, G. Kouroupetroglou, \& G. Carayiannis (Eds.), Intonation: Theory, Models, and Applications (Proceedings of an ESCA Workshop), Athens, Greece, (pp. 67-70).

Cohen, J., \& Polich, J. (1997). On the number of trials needed for P300. International Journal of Psychophysiology, 25, 249-255.

Cole, J., Mo, Y., \& Hasegawa-Johnson, M. (2010). Signal-based and expectation-based factors in the perception of prosodic prominence. Laboratory Phonology, 1, 425-452.

Connolly, J. F., \& Phillips, N. A. (1994). Event-related potential components reflect phonological and semantic processing of the terminal word of spoken sentences. Journal of Cognitive Neuroscience, 6, 256-266.

Cooper, R. P., \& Aslin, R. N. (1994). Developmental differences in infant attention to the spectral properties of infant-directed speech. Child Development, 65, 1663-1677.

Cutler, A., \& Foss, D. J. (1977). On the role of sentence stress in sentence processing. Language and Speech, 20, 1-10.

Cutler, A., Dahan, D., \& van Donselaar, W. (1997). Prosody in the comprehension of spoken language: A literature review. Language and Speech, 40, 141-201.

Cutler, A., \& Swinney, D. A. (1987). Prosody and the development of comprehension. Journal of Child Language, 14, 145-167.

Dien, J. (2017). Best practices for repeated measures ANOVA of ERP data: Reference, regional channels, and robust ANOVAs. International Journal of Psychophysiology, 111, 42-56.

Dimitrova, D. V., Stowe, L. A., Redeker, G., \& Hoeks, J. C. J. (2012). Less is not more: Neural responses to missing and superfluous accents in context. Journal of Cognitive Neuroscience, 24, 2400-2418.

Eckstein, K., \& Friederici, A. D. (2005). Late interaction of syntactic and prosodic processes in sentence comprehension as revealed by ERPs. Cognitive Brain Research, 25, 130-143. 
Erickson, L. C., \& Thiessen, E. D. (2015). Statistical learning of language: Theory, validity and predictions of a statistical learning account of language acquisition. Developmental Review, 37 , 66-108.

Escera, C., Alho, K., Winkler, I., \& Näätänen, R. (1998). Neural mechanisms of involuntary attention to acoustic novelty and change. Journal of Cognitive Neuroscience, 10, 590-604.

Federmeier, K. D. (2007). Thinking ahead: The role and roots of prediction in language comprehension. Psychophysiology, 44, 491-505.

Francois, C., Cunillera, T., Garcia, E., Laine, M., \& Rodriguez-Fornells, A. (2017). Neurophysiological evidence for the interplay of speech segmentation and word-referent mapping during novel word learning. Neuropsychologia, 98, 56-67.

Friederici, A. D. (1995). Time course of syntactic activation during language processing: A model based on neurophysiological and neurophysiological data. Brain and Language, 50, 259-281.

Friston, K., \& Kiebel, S. (2009). Cortical circuits for perceptual inference. Neural Networks, 22, 1093-1104.

Fry, D. B. (1955). Duration and intensity as physical correlates of linguistic stress. Journal of the Acoustical Society of America, 27, 765-768.

Fry, D. B. (1958). Experiments in the perception of stress. Language and Speech, 1, 126-152.

Garrido, M. I., Kilner, J. M., Stephan, K. E., \& Friston. K. J. (2009). The mismatch negativity: A review of underlying mechanisms. Clinical Neurophysiology, 120, 453-463.

Gonsalvez, C. J., \& Polich, J. (2002). P300 amplitude is determined by target-to-target interval. Psychophysiology, 39, 388-396.

Gouvea, A. C., Phillips, C., Kazanina, N., \& Poeppel, D. (2010). The linguistic processes underlying the P600. Language and Cognitive Processes, 25, 149-188.

Grossberg, S. (1987). Competitive learning: From interactive activation to adaptive resonance. Cognitive Science, 11, 23-63.

Hagoort, P. (2003). How the brain solves the binding problem or language: a neurocomputational model of syntactic processing. NeuroImage, 20, S18-29.

Hart, J. (1981). Differential sensitivity to pitch distance, particularly in speech. Journal of the Acoustical Society of America, 69, 811-821. 
Hermes, D. J., \& Rump, H. H. (1994). Perception of prominence in speech intonation induced by rising and falling pitch movements. Journal of the Acoustical Society of America, 96, 83-92.

Hruska, C., Alter, K., Steinhauer, K., \& Steube, A. (2001). Misleading dialogues: Human's brain reaction to prosodic information. In C. Cave, I. Guaitella, \& S. Santi (Eds.), Orality and gestures. Interactions et comportements multimodaux dans la communication. Paris: L'Hartmattan.

Holcomb, P. J., \& Neville, H. J. (1990). Auditory and visual semantic priming in lexical decision: a comparison using event-related brain potentials. Language and Cognitive Processes, 5, 281312.

Honbolygó, F., Török, Á., Bánreti, Z., Hunyadi, L., \& Csépe, V. (2016). ERP correlates of prosody and syntax interaction in case of embedded sentences. Journal of Neurolinguistics, 37, 22-33.

Itti, L., \& Baldi, P. (2009). Bayesian surprise attracts human attention. Vision Research, 49, 12951306.

Jurafsky, D. (1996). A probabilistic model of lexical and syntactic access and disambiguation. Cognitive Science, 20, 137-194.

Jurafsky, D., Bell, A., Gregory, M., \& Raymond, W. D. (2001). Probabilistic relations between words: Evidence from reduction in lexical production. Typological studies in language, 45, 229-254.

Kaan, E., \& Swaab, T. Y. (2003). Repair, revision, and complexity in syntactic analysis: An elecrophysiological differentiation. Journal of Cognitive Neuroscience, 15, 98-110.

Kakouros, S., \& Räsänen, O. (2014). Statistical Unpredictability of F0 Trajectories as a Cue to Sentence Stress. Proceedings of the 36th Annual Conference of the Cognitive Science Society, Quebec, Canada (pp. 1246-1251). Austin, TX: Cognitive Science Society.

Kakouros, S., \& Räsänen, O. (2016a). Perception of sentence stress in speech correlates with the temporal unpredictability of prosodic features. Cognitive Science, 40, 1739-1774.

Kakouros S., \& Räsänen O. (2016b). 3PRO - An unsupervised method for the automatic detection of sentence prominence in speech. Speech Communication, 82, 67-84.

Kakouros S., Pelemans J., Verwimp L., Wambacq P., \& Räsänen O. (2016). Analyzing the contribution of top-down lexical and bottom-up acoustic cues in the detection of sentence prominence. Proceedings of Interspeech-2016, San Francisco, CA (pp. 1074-1078). 
Kiefer, M. (2002). The N400 is modulated by unconsciously perceived masked words: further evidence from an automatic spreading activation account of N400 priming effects. Cognitive Brain Research, 13, 27-39.

Kidd, C., Piantadosi, S. T., \& Aslin, R. N. (2012). The Goldilocks effect: human infants allocate attention to visual sequences that are neither too simple nor too complex. PLoS ONE, 7(5): e36399. doi:10.1371/journal.pone.0036399.

Kidd, C., Piantadosi, S. T., \& Aslin, R. N. (2014). The Goldilocks Effect in Infant Auditory Attention. Child Development, 85, 1795-1804.

Kleinschmidt, D. F., \& Jaeger, F. (2015). Robust speech perception: recognize the familiar, generalize to the similar, and adapt to the novel. Psychological Review, 122, 148-203.

Kochanski, G., Grabe, E., Coleman, J., \& Rosner, B. (2005). Loudness predicts prominence: Fundamental frequency lends little. Journal of the Acoustical Society of America, 118, 10381054.

Koelsch, S., Busch, T., Jentschke, S., \& Rohrmeier, M. (2016). Under the hood of statistical learning: A statistical MMN reflects the magnitude of transitional probabilities in auditory sequences. Scientific Reports, 6:19741, DOI: 10.1038/srep19741.

Krishnan, A., Gandour, J. T., Ananthakrishnan, S., \& Vijayaraghavan, V. (2015). Language experience enhances early cortical pitch-dependent responses. Journal of Neurolinguistics, 33, $128-148$.

Kutas, M., \& Federmeier, K. D. (2011). Thirty years and counting: finding meaning in the N400 component of the event-related brain potential (ERP). Annual Review of Psychology, 62, 621647.

Kutas, M., \& Hillyard, S. A. (1980). Reading senseless sentences: brain potentials reflect semantic incongruity. Science, 207, 203-205.

Kutas, M., \& Van Petten, C. K. (1994). Psycholinguistics electrified: event-related brain potential investigations. In M. A. Gernsbacher (Ed.), Handbook of Psycholinguistics (pp. 83-143). San Diego, CA: Academic Press Inc.

Laszlo, S., \& Federmeier, K. D. (2011). The N400 as a snapshot of interactive processing: evidence from regression analyses of orthographic neighbor and lexical associate effects. Psychophysiology, 48, 176-186. 
Li, X., \& Ren, G. (2012). How and when accentuation influences temporally selective attention and subsequent semantic processing during on-line spoken language comprehension: An ERP study. Neuropsychologia, 50, 1882-1894.

Lieberman, P. (1960). Some acoustic correlates of word stress in American English. Journal of the Acoustical Society of America, 32, 451-454.

Magne, C., Astèsano, C., Lacheret-Dujour, A., Morel, M., Alter, K., \& Besson, M. (2005). On-line processing of "pop-out” words in spoken French dialogues. Journal of Cognitive Neuroscience, $15,740-756$.

Maier, A. K., Hönig, F., Zeißler, V., Batliner, A., Körner, E., Yamanaka, N., \& Nöth, E. (2009). A language-independent feature set for the automatic evaluation of prosody. Proceedings of Interspeech, Brighton, United Kingdom (pp. 600-603).

Maye, J., Werker, J. F., \& Gerken, L. (2002). Infant sensitivity to distributional information can affect phonetic discrimination. Cognition, 82, B101-B111.

Mietz, A., Toepel, U., Ischebeck, A., \& Alter, K. (2008). Inadequate and infrequent are not alike: ERPs to deviant prosodic patterns in spoken sentence comprehension. Brain and Language, 104, 159-169.

Moore, C. B. (1993). Phonetic observations on stress and tones in Mandarin Chinese. Working Papers of Cornell Phonetics Laboratory, 8, 89-117.

Murray, M. M., Brunet, D., \& Christoph, M. M. (2008). Topographic ERP analyses: a step-by-step tutorial review. Brain Topography, 20, 249-264.

Näätänen, R., \& Winkler, I. (1999). The concept of auditory stimulus representation in cognitive neuroscience. Psychological Bulletin, 125, 826-859.

Näätänen, R., Paavilainen, P., Rinne, T., \& Alho. K. (2007). The mismatch negativity (MMN) in basic research of central auditory processing: A review. Clinical Neurophysiology, 118, 25442590 .

Näätänen, R., Lehtokoski, A., Lennes, M., Cheour-Luhtanen, M., Huotilainen, M., Iivonen, A., Vainio, M., Alku P., Ilmoniemi, R., Luuk, A., Allik, J., Sinkkonen, J., \& Alho, K. (1997). Languge-specific phoneme representations revealed by electric and magnetic brain responses. Nature, 385, 432-434.

Ortega-Llebaria, M., \& Prieto, P. (2010). Acoustic correlates of stress in central Catalan and Castilian Spanish. Language and Speech, 54, 1-25. 
Osterhout, L., \& Holcomb, P. J. (1992). Event-related brain potentials elicited by syntactic anomaly. Journal of Memory and Language, 31, 785-806.

Pan, S., \& Hirschberg, J. (2000). Modeling local context for pitch accent prediction. Proceedings of the 38th Annual Meeting of the Association for Computational Linguistics, Hong Kong (pp. 233-240).

Pan, S., \& McKeown, K. (1999). Word informativeness and automatic pitch accent modeling. Proceedings of EMNLP/VLC, College Park, MD, USA (pp. 148-157).

Pannekamp, A., van der Meer, E., \& Toepel, U. (2011). Context- and prosody-driven ERP markers for dialog focus perception in children. Brain Topography, 24, 229-242.

Picton, T. W. (1992). The P300 wave of the human event-related potential. Journal of Clinical Neurophysiology, 9, 456-479.

Polich, J. (2007). Updating P300: An integrative theory of P3a and P3b. Clinical Neurophysiology, $118,2128-2148$.

Ranganath, C., \& Rainer, G. (2003). Neural mechanisms for detecting and remembering novel events. Nature Reviews Neuroscience, 4, 193-202.

Rietveld, A. C. M., \& Gussenhoven, C. (1985). On the relation between pitch excursion size and prominence. Journal of Phonetics, 13, 299-308.

Rosenberg, A., Cooper, E. L., Levitan, R., \& Hirschberg, J. B. (2012). Cross-language prominence detection. Proceedings of Speech Prosody, Shanghai, China (pp. 278-281).

Räsänen, O., Kakouros, S., \& Soderstrom, M. (2017). Connecting stimulus-driven attention to the properties of infant-directed speech - Is exaggerated intonation also more surprising? Proceedings of the 39th Annual Conference of the Cognitive Science Society, London, UK (pp. 998-1003).

Räsänen, O., Kakouros, S., \& Soderstrom, M. (submitted). Is infant-directed speech interesting because it is surprising? - Linking properties of IDS to statistical learning and attention at the prosodic level. Submitted for publication.

Saffran, J. R., Aslin, R. N., \& Newport, E. L. (1996). Statistical learning by 8-month-old infants. Science, 274, 1926-1928. 
Saffran, J. R., Johnson, E. K., Aslin, R. N., \& Newport, E. L. (1999). Statistical learning of tone sequences by human infants and adults. Cognition, 70, 27-52.

Samuel, A. G. (1987). Lexical uniqueness effects on phonemic restoration. Journal of Memory and Language, 26, 36-56.

Sanford, A. J. S., Sanford, A. J., Molle, J., \& Emmot, C. (2006). Shallow processing and attention capture in written and spoken discourse. Discourse Process, 42, 109-130.

Shannon, C. E. (1948). A Mathematical Theory of Communication. The Bell System Technical Journal, 27, 379-423.

Shattuck-Hufnagel, S., \& Turk, A. E. (1996). A prosody tutorial for investigators of auditory sentence processing. Journal of psycholinguistic research, 25, 193-247.

Sluijter, A. M. C., \& van Heuven, V. J. (1996). Spectral balance as an acoustic correlate of linguistic stress. Journal of the Acoustical Society of America, 100, 2471-2485.

Soderstrom, M. (2007). Beyond babytalk: Re-evaluating the nature and content of speech input to preverbal infants. Developmental Review, 27, 501-532.

Steinhauer, K., Alter, K., \& Friederici, A. D. (1999). Brain potentials indicate immediate use of prosodic cues in natural speech processing. Nature Neuroscience, 2, 191-196.

Suomi, K., Toivanen, J., \& Ylitalo, R. (2008). Finnish sound structure: Phonetics, phonology, phonotactics and prosody. University of Oulu, Oulu, Finland.

Suomi, K., \& Ylitalo, R. (2002). Word stress and duration in Finnish. In C. Dunger, B. Granström, D. House, \& A. M. Öster (Eds.), Proceedings of the Swedish Phonetics Conference (Fonetik2002) (pp. 73-76).

Terken, J. (1991). Fundamental frequency and perceived prominence of accented syllables. Journal of the Acoustical Society of America, 89, 1768-1776.

Terken, J., \& Nooteboom, S. G. (1987). Opposite effects of accentuation and deaccentuation on verification latencies for given and new information. Language and cognitive processes, 2(34), 145-163.

Tervaniemi, M., Maury, S., \& Näätänen, R. (1994). Neural representations of abstract stimulus features in the human brain as reflected by the mismatch negativity. NeuroReport, 5, 844-846. 
Toepel, U., \& Alter, K. (2004). On the independence of information structure processing from prosody. In A. Stuebe (Ed.), Information structure: Theoretical and empirical evidence (pp. 227-240). Berlin: Walter de Gruyter.

Toepel, U., Pannekamp, A., \& Alter, K. (2007). Catching the news: Processing strategies in listening to dialogs as measured by ERPs. Behavioral and Brain Functions, 3:53, DOI: 10.1186/1744-9081-3-53.

Tong, X., McBride, C., Zhang, J., Chung, K. K. H., Lee, C-Y., Shuai, L., \& Tong X. (2014). Neural correlates of acoustic cues of English lexical stress in Cantonese-speaking children. Brain \& Language, 138, 61-70.

Treisman, A. (1964). Monitoring and storage of irrelevant messages in selective attention. Journal of Verbal Learning and Verbal Behavior, 3, 449-459.

Tsuchida, T., \& Cottrell, G. W. (2012). Auditory saliency using natural statistics. Proceedings of the 34th Annual Conference of the Cognitive Science Society (CogSci-2012), Sapporo, August 1-4 (pp. 1048-1053).

Vaissière, J. (1983). Language-independent prosodic features. In A. Cutler \& D. R. Ladd (Eds.), Prosody: Models and measurements (pp. 53-66). Berlin Heidelberg: Springer.

Van Berkum, J. J. A. (2009). The neuropragmatics of 'simple' utterance comprehension: An ERP review. In Sauerland, U., \& Yatsushiro, K. (Eds.): Semantics and pragmatics: From experiment to theory (pp. 276-316). Basingstoke: Palgrave Macmillan.

van den Brink, D., Brown, C. M., \& Hagoort, P. (2001). Electrophysiological evidence for early contextual influences during spoken-word recognition: N200 versus N400 effects. Journal of Cognitive Neuroscience, 13, 967-985.

Van Petten, C., Coulson, S., Rubin, S., Plante, E., \& Parks, M. (1999). Time course of word identification and semantic integration in spoken language. Journal of Experimental Psychology: Learning, Memory and Cognition, 25, 394-417.

Wagner, P., Origlia, A., Avesani, C., Christodoulides, G., Cutugno, F., D'Imperio, M., ... \& Vainio, M. (2015). Different Parts of the Same Elephant: a Roadmap to Disentangle and Connect Different Perspectives on Prosodic Prominence. In The Scottish Consortium for ICPhS 2015 (Ed.), Proceedings of the 18th International Congress of Phonetic Sciences (ICPhS-2015). Glasgow, UK: the University of Glasgow. 
Wang, L., Bastiaansen, M., Yang, Y., \& Hagoort, P. (2011). The influence of information structure on the depth of semantic processing: How focus and pitch accent determine the size of the N400 effect. Neuropsychologia, 49, 813-820.

Warren, R. M. (1970). Perceptual restoration of missing speech sounds. Science, 167, 329-393.

Watson, D. G., Arnold, J. E., \& Tanenhaus, M. K. (2008). Tic Tac TOE: Effects of predictability and importance on acoustic prominence in language production. Cognition, 106, 1548-1557.

Winkler, I., Cowan, N., Csépe, V., Czigler, I., \& Näätänen, R. (1996). Interactions between transient and long-term auditory memory as reflected by the mismatch negativity. Journal of Cognitive Neuroscience, 8, 403-415.

Woodman, G. F. (2010). A brief introduction to the use of event-related potentials (ERPs) in studies of perception and attention. Attention, Perception \& Psychophysics, 72(8), 2031-2046.

Ylitalo, R. (2009). The realization of prominence in three varieties of standard spoken Finnish. Doctoral thesis, University of Oulu, Faculty of Humanities, Finland.

Zarcone, A., van Schijndel, M., Vogels, J., \& Demberg, V. (2016). Salience and attention in surprisal-based accounts of language processing. Frontiers in Psychology, 7, article no. 844.

Zhang, L., Tong, M. H., Marks, T. K., Shan, H., \& Cottrell, G. W. (2008). SUN: A Bayesian framework for saliency using natural statistics. Journal of Vision, 8, 1-20. 


\section{Appendix A: Effects of F0 direction in experiment 2}

Since both falling and rising F0 were used as standards and deviants in the experiment 2, we also tested whether significant differences in responses to these two types of F0 patterns were observable in the measured EEG data. Table A1 shows the post-hoc comparisons between rising and falling F0 stimuli across each combination of time window and electrode, following the same statistical testing procedure used in Table 1 to report the effects of condition (t-test, HolmBonferroni correction). No statistically significant differences were observed between rising and falling F0.

Table A1: Pair-wise post-hoc test results (unpaired t-test) between rising and falling F0 stimuli for each time window and electrode using pooled responses across all trials and subjects. Adjusted p-values are reported with Holm-Bonferroni multiple-comparisons correction for $p<0.05$ and with $d f=15$ for all tests.

\begin{tabular}{|c|c|c|c|c|c|c|c|c|}
\hline channel & \multicolumn{2}{|c|}{ F3+F4 } & \multicolumn{2}{c|}{ Fz } & \multicolumn{2}{c|}{ FC1+FC2 } & \multicolumn{2}{c|}{ C3+C4 } \\
\hline time (ms) & $p$ & $t$ & $p$ & $t$ & $p$ & $t$ & $p$ & $t$ \\
\hline $\mathbf{1 5 0 - 3 0 0}$ & 0.685 & 2.501 & 1.000 & 2.043 & 1.000 & 2.002 & 1.000 & 1.327 \\
\hline $\mathbf{2 0 0 - 4 5 0}$ & 0.130 & 3.373 & 0.101 & 3.512 & 0.841 & 2.378 & 1.000 & 1.178 \\
\hline $\mathbf{3 0 0 - 5 5 0}$ & 1.000 & 0.585 & 1.000 & 0.578 & 1.000 & 1.233 & 1.000 & 0.657 \\
\hline $\mathbf{5 0 0 - 8 0 0}$ & 1.000 & 0.695 & 1.000 & 0.560 & 1.000 & 0.470 & 1.000 & 0.540 \\
\hline channel & \multicolumn{2}{|c|}{$\mathrm{Cz}$} & \multicolumn{2}{|c|}{$\mathrm{CP1+CP2}$} & \multicolumn{2}{|c|}{$\mathbf{P z}$} & \multicolumn{2}{|c|}{ P3+P4 } \\
\hline time (ms) & $p$ & $t$ & $p$ & $t$ & $p$ & $t$ & $p$ & $t$ \\
\hline $\mathbf{1 5 0 - 3 0 0}$ & 1.000 & 0.413 & 1.000 & 1.274 & 1.000 & 2.090 & 0.843 & 2.357 \\
\hline $\mathbf{2 0 0 - 4 5 0}$ & 1.000 & 0.601 & 1.000 & 1.370 & 0.672 & 2.528 & 0.462 & 2.733 \\
\hline $\mathbf{3 0 0 - 5 5 0}$ & 1.000 & 1.739 & 1.000 & 0.833 & 1.000 & 0.456 & 1.000 & 0.181 \\
\hline $\mathbf{5 0 0 - 8 0 0}$ & 1.000 & 0.444 & 1.000 & 0.610 & 1.000 & 0.908 & 1.000 & 0.837 \\
\hline
\end{tabular}

Figure A1 shows the difference response between deviant and standard conditions separately for the rising and falling F0 in case of electrodes with significant differences for the main experimental condition. As can be observed from the figure, the same ERP components (N200 and N400; latter reflected as positive waveform at frontal sites) are visible in case of both types of prosodic trajectories, indicating that the direction of F0 change was is not a major factor in determining the responses.
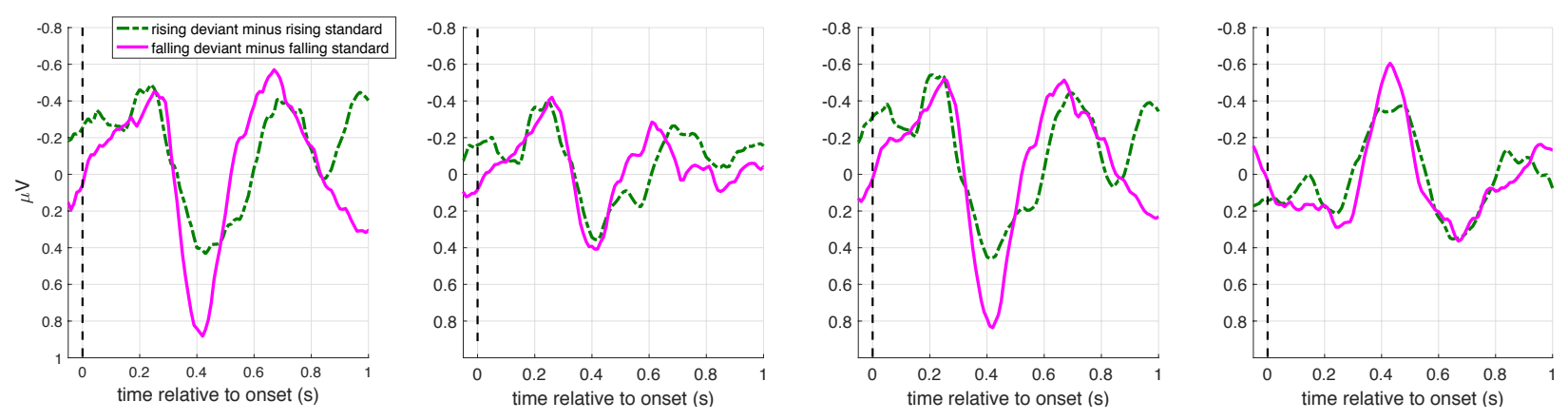

Figure A1: Grand-average differential waveforms (deviants minus standards) for rising F0 stimuli (dashed green line) and falling F0 stimuli (solid magenta line). 


\section{Appendix B: Results of experiment 2 with artifact filtering}

Table B1 shows the difference between standard and deviant conditions in experiment 2 when all trials exceeding $\pm 50 \mu \mathrm{V}$ in signal amplitude are excluded from the analyses.

Table B1: Pair-wise post-hoc test results (paired t-test) between conditions for each time window and electrode channel using subject average responses for each condition and using artifact filtering threshold $\pm 50 \mu \mathrm{V}$. Adjusted p-values are reported with Holm-Bonferroni multiple-comparisons correction for $p<0.05$ and with $d f=15$ for all tests.

\begin{tabular}{|c|c|c|c|c|c|c|c|c|}
\hline channel & \multicolumn{2}{|c|}{ F3+F4 } & \multicolumn{2}{c|}{ Fz } & \multicolumn{2}{c|}{ FC1+FC2 } & \multicolumn{2}{c|}{ C3+C4 } \\
\hline time (ms) & $p$ & $t$ & $p$ & $t$ & $p$ & $t$ & $p$ & $t$ \\
\hline $\mathbf{1 5 0 - 3 0 0}$ & 0.232 & 2.874 & 0.166 & 3.084 & $\mathbf{0 . 0 0 6 *}$ & $\mathbf{4 . 9 0 7 *}$ & 0.712 & 2.123 \\
\hline $\mathbf{2 0 0 - 4 5 0}$ & 1.000 & 0.602 & 1.000 & 0.682 & 1.000 & 0.514 & 0.546 & 2.331 \\
\hline $\mathbf{3 0 0 - 5 5 0}$ & $\mathbf{0 . 0 3 5 *}$ & $\mathbf{3 . 9 8 3 *}$ & $\mathbf{0 . 0 1 7}$ & $\mathbf{4 . 3 6 1 *}$ & $\mathbf{0 . 0 0 8 *}$ & $\mathbf{4 . 7 6 7 *}$ & 0.530 & 2.434 \\
\hline $\mathbf{5 0 0 - 8 0 0}$ & 0.060 & 3.680 & 0.110 & 3.347 & 0.539 & 2.369 & 0.781 & 2.033 \\
\hline channel & \multicolumn{2}{|c|}{$\mathrm{Cz}$} & \multicolumn{2}{|c|}{$\mathrm{CP1+CP2}$} & \multicolumn{2}{|c|}{$\mathrm{Pz}$} & \multicolumn{2}{|c|}{ P3+P4 } \\
\hline time (ms) & $p$ & $t$ & $p$ & $t$ & $p$ & $t$ & $p$ & $t$ \\
\hline $\mathbf{1 5 0 - 3 0 0}$ & 0.530 & 2.415 & 1.000 & 0.323 & 1.000 & 1.445 & 1.000 & 1.689 \\
\hline $\mathbf{2 0 0 - 4 5 0}$ & 1.000 & 0.982 & 1.000 & 1.548 & 1.000 & 1.260 & 1.000 & 0.873 \\
\hline $\mathbf{3 0 0 - 5 5 0}$ & 1.000 & 0.363 & 0.201 & 2.969 & 0.090 & 3.467 & $\mathbf{0 . 0 3 7}$ & $\mathbf{3 . 9 3 9 *}$ \\
\hline $\mathbf{5 0 0 - 8 0 0}$ & 1.000 & 0.442 & 0.613 & 2.238 & 0.133 & 3.214 & 0.122 & 3.278 \\
\hline
\end{tabular}

